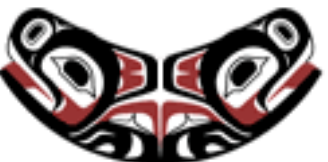

UW Biostatistics Working Paper Series

6-9-2009

\title{
Evaluating Markers for Treatment Selection Based on Survival Time
}

\author{
Xiao Song \\ University of Georgia, Athens, xsong.uga@gmail.com \\ Xiao-Hua Zhou \\ University of Washington, azhou@u.washington.edu
}

\section{Suggested Citation}

Song, Xiao and Zhou, Xiao-Hua , "Evaluating Markers for Treatment Selection Based on Survival Time" (June 2009). UW Biostatistics Working Paper Series. Working Paper 349.

http://biostats.bepress.com/uwbiostat/paper349

This working paper is hosted by The Berkeley Electronic Press (bepress) and may not be commercially reproduced without the permission of the copyright holder.

Copyright (c) 2011 by the authors 


\title{
Evaluating Markers for Treatment Selection Based on Survival Time
}

\author{
Xiao Song \\ University of Georgia, Athens, USA
}

Xiao-Hua Zhou

University of Washington and Puget Sound Health Care System, Seattle, USA

\begin{abstract}
Summary.
For many medical conditions several treatment options may be available for treating patients. We consider evaluating markers based on a simple treatment selection policy that incorporates information on the patient's marker value exceeding a threshold. For example, colon cancer patients may be treated by surgery alone or surgery plus chemotherapy. The c-myc gene expression level may be used as a biomarker for treatment selection. Although traditional regression methods may assess the effect of the marker and treatment on outcomes, it is appealing to quantify more directly the potential impact on the population of using the marker to select treatment. A useful tool is the selection impact (SI) curve proposed by Song and Pepe (2004, Biometrics 60, 874-883) for binary outcomes. However, the current SI method does not deal with continuous outcomes, nor does it allow to adjust for other covariates that are important for treatment selection. In this paper, we extend the SI curve for general outcomes, with a specific focus on survival time. We further propose the covariate specific SI curve to incorporate covariate information in treatment selection. Nonparametric and semiparametric estimators are developed accordingly. We show that the proposed estimators are consistent and asymptotically normal. The performance is illustrated by simulation studies and through an application to data from a cancer clinical trial.
\end{abstract}

\section{INTRODUCTION}

Selecting an appropriate treatment for patients is important when several treatment options are available. Treatment selection may be facilitated by evaluating clinical characteristics or biomarker measurements of patients at diagnosis. The rapidly expanding biotechnologies, including gene expression arrays and imaging modalities, show promises in providing useful biomarkers that may be used for selection of the optimal treatment of disease (Elmer-Dewitt et al., 2001). For example, patients with colon cancer can be treated by surgery alone or surgery plus chemotherapy. Surgery alone is less invasive and less expensive than surgery plus chemotherapy, but it may be less effective as well, at least for some patients. It is desirable to identify the patients who may benefit more from surgery based on biomarkers. A possible useful biomarker is the c-myc gene, which is overexpressed in approximately $70 \%$

Address for correspondence: Xiao Song, Department of Biostatistics and Epidemiology, College of Public Health, University of Georgia, Paul Coverdell Center, Room 129c, Athens 30602, USA. E-mail: xsong@uga.edu 
of human colonic tumors (Erisman et al., 1988). Based on a study conducted by the Eastern Cooperative Oncoloty Group (ECOG), Augenlicht et al. (1997) suggested that the c-myc gene may be of clinical prognostic importance in patients with colon cancer. Using a subset of the cases from this clinical trial, Li and Ryan (2006) indicated that there is an interaction between the c-myc gene expression level and the two treatments on overall survival and disease progression free survival. Using the same dataset, we estimated the overall survival and the disease progression free survival by treatment and c-myc gene expression level. Figure 1 shows the Kaplan-Meier estimates of the disease progression survival for the four combinations of the two treatments and whether the c-myc gene expression level exceeding 1.05 , the $25 \%$ sample percentile. Although surgery plus chemotherapy seems prolonging disease progression free survival better than surgery alone for patients with c-myc level > 1.05 , it is not that clear which treatment is better for patients with c-myc level $\leq 1.05$. The Kaplan-Meier estimates for the overall survival show a similar pattern (not shown). This motivated us to assess using the c-myc gene expression level for treatment selection for colon cancer patients.

In clinical protocols, treatment selection is often based on whether a marker value exceeds a threshold. Some common examples include serum creatine $>1.3 \mathrm{mg} / \mathrm{dL}$, cholesterol $>200 \mathrm{mg} / \mathrm{dL}$, and serum PSA > $4.0 \mathrm{ng} / \mathrm{mL}$. As an analogy, we consider selection of surgery alone versus chemotherapy plus surgery based on c-myc level exceeding a threshold. To evaluate such policies, Song and Pepe (2004) proposed a graphical tool, the selection impact (SI) curve, for selection between two treatments based on binary response rate using a biomarker. Let $A=0$ denote one treatment and $A=1$ denote the other. The outcome $T$ is dichotomous denoting success $(T=1)$ or failure $(T=0)$ in curing a disease. Let $Y$ be a continuous biomarker and larger values of $Y$ are potentially associated with better performance of treatment 1 versus 0 ( $Y$ can be recoded if necessary to achieve this ). Consider the following treatment policy that determines which treatment the patient receives based on a patient's marker measure $Y$ exceeding a threshold:

$$
\begin{array}{ll}
\text { if } Y>c, & \text { select treatment } A=1 ; \\
\text { if } Y \leq c, & \text { select treatment } A=0,
\end{array}
$$

The population response (success) rate of the outcome corresponding to this policy is

$$
\theta(v)=\operatorname{Pr}\{T=1 \mid \text { treatment policy (1) }\}
$$

where $v=\operatorname{Pr}[Y \leq c]$ is the proportion of subjects with the marker value below $c$ and hence assigned to treatment 0 . That is, $\theta$ is the proportion of subjects in the population who respond if the treatment policy in effect is to assign a subject to treatment 1 if his marker value exceeds $c$ but to assign him to treatment 0 otherwise. Observe that when $c=-\infty$ or equivalently $v=0$, the policy is that all patients receive treatment 1 and none receive 0 , while for $c=\infty$ or equivalently $v=1$ all patients receive treatment 0 . As $c$ increases from $-\infty$ to $\infty$, the proportion of subjects assigned to treatment 0 increases from 0 to 1 . There are two reasons for defining $\theta$ as a function of quantile $v$. First, in evaluating a treatment policy of this sort, it will be important to know the fractions of patients potentially assigned to treatment $A=0$ versus $A=1$ by the policy, $1-v$ and $v$, respectively. Second, the display on this scale allows one to compare policies based on different markers. In particular, even if the markers are measured in different units, we can still compare the treatment policies at a common percentile $v$, the larger $\theta(v)$ the better. The same idea has been adopted for the receiver operative curve (ROC), which is widely used in evaluation of diagnostic tests 
(Zhou, et al., 2002; Pepe, 2003). When the two treatments are comparable in all other aspects including cost and side effects, then the optimal threshold maximizes the SI curve $\theta(v)$ such that the overall success rate achieves the maximum. As illustrated in Figure 2, based on the biomarker $Y_{1}$, the best criteria would be to assign $40 \%$ patients to treatment 0 whose biomarker value is less than the $40 \%$ percentile, while $Y_{3}$ indicates it would be best to assign all patients to treatment 1 . Based on the biomarker $Y_{2}$, for any $v$ between 0 and 0.4 , the success rate would be the same if we assign patients to treatment 0 whose biomarker value falls below the $v$ th percentile. However, if treatment 1 is more invasive or more expensive, we may want to assign $40 \%$ patients to treatment 0 such that the overall success rate achieves the maximum. Comparing the three biomarkers, it is obviously that policy based on $Y_{1}$ achieves the best success rate.

The SI curve is essentially a curve of the population response rate versus the percentile threshold. Compared to traditional regression models, this approach has the advantage of quantifying more directly the potential impact on the population of using the biomarker to select treatment. Specifically, we may choose an optimal threshold based on the the SI curve. However, there is a need for further improvement for wider applicability. First, the current SI curve methodology considers only binary outcomes. It cannot be applied to continuous outcomes such as survival time, which is frequently encountered in practice. For example, in the ECOG study (Li and Ryan, 2006), the outcome of interest is the overall survival time and the disease progression free survival of the colon cancer patients. It may sometimes be desirable to select treatment based on a utility measure that incorporates notions of cost and benefit when those factors differ for the two treatments. Second, the current SI curve does not allow to adjust for other covariates, which may contain additional important information for treatment selection. For colon cancer patients, the c-myc gene may be indicative for which patients are likely to benefit more from surgery alone. The effect of c-myc gene may be further impacted by covariates like gender and stage of cancer. For example, the optimal threshold for c-myc gene expression level may be different for patients with different covariate values. For example, Figures 3(a) and (b) show the Kaplan-Meier estimates of disease progression free survival by treatment and c-myc gene expression level above or below 1.05 for females and males separately. For either females or males with c-myc level $>1.05$, surgery plus chemotherapy seems better than surgery alone in terms of prolonging disease progression free survival. However, for patients with c-myc level $\leq 1.05$, females seem benefited more from surgery while it is not clear which treatment is better for males. In addition, the SI curve methodology was not applied to any real dataset in Song and Pepe (2004). It is of great interest to demonstrate this method in real applications such as the ECOG study.

To overcome the limitations of the current SI curve methodology, we generalize the current SI curve definition in two steps. First, we propose the SI curve for general outcomes for evaluating markers on treatment selection; Second, we extend the SI curve to adjust for covariates. In this paper, we focus specifically on survival time, which is the outcome of interest in the ECOG data and is more challenging compared to discrete and continuous outcomes without censoring. The same technique can be easily adapted to the latter with only minor modifications. We propose the SI curve based on the mean restricted survival time up to a given time $L$. The reason of using mean restricted survival time is to avoid the infeasibility of estimating the mean unrestricted survival time when censoring exists, that is, the mean unrestricted survival time may not be estimated if the largest observed survival time is censored without some tail correction on the estimated survival function (Klein and Moeschberger, 2003). The technique of restricting survival time has been used Resecroh Archive 
previously in estimating the mean lifetime and quality-adjusted lifetime (e.g., Zhao and Tsiatis, 1999; Chen and Tsiatis, 2001). The restricted survival time has also been widely used in practice, for example, in cancer statistics, five year survival has been commonly used. Due to the existence of censoring, the inference is more challenging than the binary case. A nonparametric estimator is proposed to estimate the SI curve with no model assumptions on survival time. To adjust for covariate effects, we further propose the covariate specific SI curve and develop semiparametric estimators based on the proportional hazards model. Asymptotic properties of the estimators are derived using empirical process and U-process theories. The approach can be adapted to uncensored continuous outcomes with some modifications.

This paper is organized as follows. In Section 2, we define the SI curve for survival time. develop the nonparametric estimator and derive its asymptotic properties. In Section 3, we define the covariate specific SI curve. We further derive the semiparametric estimators and their asymptotic properties. The finite sample performance of these estimators is assessed via simulation studies in Section 4 and illustrated by application to the ECOG data in Section 5. The paper concludes with discussions in Section 6.

\section{SELECTION IMPACT CURVE}

\subsection{Definition}

We extend the SI curve to a general outcome $T$, that is, $T$ can be either discrete or continuous. We define the SI curve as

$$
\theta(v)=E\{T \mid \text { treatment policy (1) }\}
$$

Here $v=\operatorname{Pr}[Y \leq c]$ is the proportion of patients assigned to treatment 0 under policy (1). The SI curve considered in Song and Pepe (2004) is a special case of (2) when $T$ is dichotomous. In this paper, we focus on SI curve for survival time hereafter.

Using the notion of potential responses (Rubin 1974, 1978; Holland, 1986), for $a=0,1$, we define $T^{a}$ as the survival time if a patient receives treatment $A=a$. It is impossible to observe $T^{0}$ and $T^{1}$ on the same patient; instead, we can only observe $T=A T^{1}+(1-A) T^{0}$. Let $T^{* a}=\min \left(T^{a}, L\right)$, be the corresponding restricted survival time by time $L$. Similarly, $T^{* 0}$ and $T^{* 1}$ can not be observed on the same patient and only $T^{*}=A T^{* 1}+(1-A) T^{* 0}$ is observable. Both $T$ and $T^{*}$ are subject to censoring. We define the SI curve as the mean restricted survival time corresponding policy (1), that is,

$$
\begin{aligned}
\theta(v) & =E\left\{T^{*} \mid \text { treatment policy }(1)\right\} \\
& =(1-v) E\left\{T^{* 1} \mid Y>y_{v}\right\}+v E\left\{T^{* 0} \mid Y \leq y_{v}\right\} .
\end{aligned}
$$

When the two treatments are comparable, the optimal threshold maximizes $\theta(v)$. Otherwise, a utility measure incorporating the survival time, cost and side effects can be used. We illustrate an application of a simple utility measure in Section 5.

\subsection{Estimation}

We now consider estimation of $\theta(v)$ using data obtained from a randomized trial where the failure time is subject to censoring. Let $C$ denote the censoring time. The observed survival data consist of $X=\min (T, C)$ and $\Delta=I(T \leq C)$. Suppose the observations 
$\left(X_{i}, \Delta_{i}, Y_{i}, A_{i}\right), i=1, \ldots, n$, are an i.i.d. sample from the distribution of $(X, \Delta, Y, A)$. We make the following assumptions: (i) patients enrolled in the trial are a simple random sample from the population of interest; (ii) the treatment assignment for patients in the trial does not depend on his marker value, that is, $A_{i}$ and $Y_{i}$ are independent.

Note that (2) can be rewritten as

$$
\theta(v)=(1-v) \int_{0}^{L} S^{(1)}\left(t, y_{v}\right) d t+v \int_{0}^{L} S^{(0)}\left(t, y_{v}\right) d t,
$$

where $S^{(1)}(t, y)=P(T \geq t \mid A=1, Y>y)$ and $S^{(0)}(t, y)=P(T \geq t \mid A=0, Y \leq y)$. Therefore, an estimator of $\theta(v)$ can be obtained by substituting estimators for $S^{(a)}(t, y)$ $(a=0,1)$ and $y_{v}$. Here we use the Kaplan-Meier estimator of $S^{(a)}(t, y)$,

$$
\hat{S}^{(a)}(t, y)=\tau_{0}^{t}\left\{1-\frac{N_{i}^{(a)}(d s, y)}{R_{i}^{(a)}(s, y)}\right\}
$$

where $\pi$ is the product integral notation, $N_{i}^{(1)}(t, y)=I\left(X_{i} \leq t, \Delta_{i}=1, A_{i}=1, Y_{i}>y\right)$, $N_{i}^{(0)}(t, y)=I\left(X_{i} \leq t, \Delta_{i}=1, A_{i}=0, Y_{i} \leq y\right), R_{i}^{(1)}(t, y)=I\left(X_{i} \geq t, A_{i}=1, Y_{i}>y\right)$, and $R_{i}^{(0)}(t, y)=I\left(X_{i} \geq t, A_{i}=0, Y_{i} \leq y\right), N_{i}^{(a)}(d s, y)=N_{i}^{(a)}(s, y)-N_{i}^{(a)}(s-, y)$. To estimate $y_{v}$, we use the nonparametric estimator $\hat{y}_{v}=\hat{F}_{Y}^{-1}(v)$, where $\hat{F}_{Y}(y)=n^{-1} \sum_{i=1}^{n} I\left(Y_{i} \leq y\right)$ is the empirical distribution of $Y$. Thus an estimator of $\theta(v)$ can be written as

$$
\hat{\theta}(v)=(1-v) \int_{0}^{L} \hat{S}^{(1)}\left(t, \hat{y}_{v}\right) d t+v \int_{0}^{L} \hat{S}^{(0)}\left(t, \hat{y}_{v}\right) d t .
$$

This is a nonparametric estimator. It does not require any parametric assumptions on the survival time and the biomarker. Moreover, it is invariant to any monotone increasing transformation of the biomarker.

Now we derive the asymptotic properties of $\hat{\theta}(v)$ using empirical process theory. The idea is to first show that $n^{1 / 2}\left\{\hat{S}^{(a)}(t, y)-S^{(a)}(t, y)\right\}$ converges to a Gaussian process under some regularity conditions. Since $\hat{\theta}(v)$ is a composite function of $\hat{S}^{(0)}, \hat{S}^{(1)}$ and $\hat{F}_{Y}$, the asymptotic distribution of $\hat{\theta}(v)$ is then derived by the functional delta method (van der Vaart and Wellner 2000, chap. 3.9). Specifically, we can show that $n^{1 / 2}\{\hat{\theta}(v)-\theta(v)\}$ converges to a Gaussian process. The details of the asymptotic distribution and the proof are given in Appendix A.

The covariance formula for $\hat{\theta}(v)$ contains the density function of $Y$ and the derivative of the cumulative hazard functions of $T$ conditional on $(A=1, Y>y)$ or $(A=0, Y \leq y)$. Usually smoothing techniques are needed to estimate these quantities. In applications, to avoid the complexity of the smoothing approaches, for simplicity, we use the bootstrap method to calculate the standard error and confidence band for $\theta(v)$. This is justified by the empirical process theory for the bootstrap given in van der Vaart and Wellner $(2000, \S 3.9 .3)$. Let $\Theta=\left\{\left(X_{i}, \Delta_{i}, Y_{i}, A_{i}\right): i=1, \ldots, n\right\}$ be the observed data set, and $\Theta_{B}$ be the $B^{\text {th }}$ resampling bootstrap dataset, where $B=1, \ldots, M$. Let $\hat{\theta}^{B}(v)$ be the estimator based on $\Theta_{B}$. Then $\sup _{v \in[p, q]}\left|\hat{\theta}^{B}(v)-\hat{\theta}(v)\right|$ given $\Theta$ is asymptotically equivalent to $\sup _{v \in[p, q]}|\hat{\theta}(v)-\theta(v)|, 0<p<q<1$. Let $c_{\alpha}$ be the $1-\alpha$ quantile of $\sup _{v \in[p, q]}\left|\hat{\theta}^{B}(v)-\hat{\theta}(v)\right|$, then a level $1-\alpha$ confidence band for $\theta(v)$ is $\left(\hat{\theta}^{B}(v)-c_{\alpha}, \hat{\theta}^{B}(v)+c_{\alpha}\right)$. The standard error of $\hat{\theta}(v)$ can be estimated by the standard deviation of $\left\{\hat{\theta}^{B}(v): B=1, \ldots, M\right\}$. 


\section{COVARIATE SPECIFIC SELECTION IMPACT CURVE}

So far, we have considered treatment selection based only on the biomarker. There are situations that covariates other than the biomarker may impact treatment selection. Thus it is important to adjust for such covariates. For example, if we use c-myc gene expression level to select patients for surgery alone or surgery plus chemotherapy, we may want to adjust for sex. If the covariates are discrete, it is possible to consider a separate SI curve for each covariate combination. However, this will not work when there exist continuous covariates. In addition, even if all covariates are discrete, the sample size for some covariate combination may be too small to obtain a reliable SI curve estimate. It is noticeable that the SI curve bears some similarities to the ROC curve used to evaluate diagnostic tests based on a biomarker (Pepe 2000). To account for covariate effects, the ROC curve has been extended to the covariate specific ROC curve (Zhou et al, 2002; Pepe, 2003). This motivates us to propose the covariate specific SI curve by analogy to the covariate specific ROC curve.

\subsection{Definition}

Let $\boldsymbol{Z}$ denote the vector of $K$ covariates that may impact the treatment selection other than the biomarker. To incorporate the covariates, we consider the following treatment policy given $\boldsymbol{Z}=\boldsymbol{z}$ :

$$
\begin{array}{ll}
\text { if } Y>y_{v, \boldsymbol{z}}, & \text { select treatment } A=1 ; \\
\text { if } Y \leq y_{v, \boldsymbol{z}} \quad, \quad \text { select treatment } A=0 .
\end{array}
$$

Here $y_{v, \boldsymbol{z}}$ is the $v$ th quantile of the conditional distribution of $Y$ given $\boldsymbol{Z}=\boldsymbol{z}$. The corresponding covariate specific SI curve is defined as

$$
\begin{aligned}
\theta(v \mid \boldsymbol{z}) & =E\left\{T^{*} \mid \text { treatment policy }(3)\right\} \\
& =(1-v) E\left\{T^{* 1} \mid Y>y_{v, \boldsymbol{z}}, \boldsymbol{Z}=\boldsymbol{z}\right\}+v E\left\{T^{* 0} \mid Y \leq y_{v, \boldsymbol{z}}, \boldsymbol{Z}=\boldsymbol{z}\right\} .
\end{aligned}
$$

This definition is general to any outcomes, include binary and continuous outcomes. It reduces to the unadjusted SI curve given in (2) when there is no covariate.

The covariate specific SI curve considers treatment selection conditional on the covariate $\boldsymbol{Z}$, which may be different for various values of $\boldsymbol{Z}$. It provides a useful tool for policy makers to choose different biomarker thresholds based on the values of $\boldsymbol{Z}$.

\subsection{Estimation}

Now we consider estimation of $\theta(v \mid \boldsymbol{z})$ using data obtained from randomized trials, which satisfies condition (i) and (ii) described in Section 2.2 and (iii) ${ }^{\prime} A$ is independent of $Y$ given $\boldsymbol{Z}$. Suppose we have independent and identically distributed observations $\left(X_{i}, \Delta_{i}, Y_{i}, A_{i}, \boldsymbol{Z}_{i}\right)$, $i=1, \ldots, n$.

With some simple algebra, it can be shown that (4) can be rewritten as

$$
\theta(v \mid \boldsymbol{z})=\int_{v}^{1} \int_{0}^{L} S(t \mid u, \boldsymbol{z}, 1) d t d u+\int_{0}^{v} \int_{0}^{L} S(t \mid u, \boldsymbol{z}, 0) d t d u
$$

where $S(t \mid u, \boldsymbol{z}, a)=\mathrm{P}\left\{T \geq t \mid Y=y_{u, \boldsymbol{z}}, \boldsymbol{Z}=\boldsymbol{z}, A=a\right\}$. An estimator of $\theta(v \mid \boldsymbol{z})$ can be obtained based on (5). 
Assume that the hazard of failure follows the proportional hazards model

$$
\lambda_{i}\left(t \mid V_{i}, \boldsymbol{Z}_{i}, A_{i}\right)=\lambda_{0}(t) \exp \left[\beta_{0}^{T} g\left\{V_{i}, \boldsymbol{Z}_{i}, A_{i}\right\}\right],
$$

where $V_{i}=F_{Y \mid \boldsymbol{Z}}\left(Y_{i} \mid \boldsymbol{Z}_{i}\right), F_{Y \mid \boldsymbol{Z}}(\cdot \mid \boldsymbol{z})$ is the conditional distribution function of $Y$ given $\boldsymbol{Z}=\boldsymbol{z}$, and $g(v, \boldsymbol{z}, a)$ is a known $r$-dimensional function. It is useful to write the model in terms of $V_{i}$, since $\theta(v)$ is considered a function of $v$ for the reasons mentioned earlier. For example we might use the model

$$
\begin{aligned}
\lambda_{i}\left(t \mid V_{i}, \boldsymbol{Z}_{i}, A_{i}\right)= & \lambda_{0}(t) \exp \left[\beta_{1} D\left(V_{i}, \boldsymbol{Z}_{i}\right)+\beta_{2}^{T} \boldsymbol{Z}_{i}+\beta_{3} A_{i}+\beta_{4} D\left(V_{i}, \boldsymbol{Z}_{i}\right) A_{i}\right. \\
& \left.+\beta_{5}^{T} D\left(V_{i}, \boldsymbol{Z}_{i}\right) Z_{i}+\beta_{6} D\left(V_{i}, \boldsymbol{Z}_{i}\right) A_{i}+\beta_{7}^{T} D\left(V_{i}, \boldsymbol{Z}_{i}\right) Z_{i} A_{i}\right],
\end{aligned}
$$

where $D(v, z)$ is a function of $v$ and $z$. When $D(v, z)=F_{Y \mid \boldsymbol{Z}}^{-1}(v \mid z), D\left(v_{i}, \boldsymbol{Z}_{i}\right)$ is just the biomarker $Y_{i}$. Further assuming that $F_{Y \mid \boldsymbol{Z}}(\cdot \mid \boldsymbol{z})$ is known, based on (5), we can estimate $\theta(v \mid \boldsymbol{z})$ by

$$
\tilde{\theta}(v \mid \boldsymbol{z})=\int_{v}^{1} \int_{0}^{L} \hat{S}(t \mid u, \boldsymbol{z}, 1) d t d u+\int_{0}^{v} \int_{0}^{L} \hat{S}(t \mid u, \boldsymbol{z}, 0) d t d u
$$

where

$$
\hat{S}(t \mid v, \boldsymbol{z}, a)=\exp \left\{-\hat{\Lambda}_{0}(t) \exp \left(\hat{\beta}^{T} g(v, \boldsymbol{z}, a)\right)\right\}
$$

is an estimator for the survival function $S(t \mid v, \boldsymbol{z}, a), \hat{\beta}$ is the partial likelihood estimator of $\beta_{0}$, and $\hat{\Lambda}_{0}(t)$ is the Breslow estimator of the baseline cumulative hazard function $\Lambda_{0}(t)$.

In practice $F_{Y \mid Z}$ is usually unknown. We assume the conditional distribution of $Y$ given $\boldsymbol{Z}$ following the semiparametric model (Pepe, 1998; Heagerty and Pepe, 1999),

$$
F_{Y \mid \boldsymbol{Z}}(y \mid \boldsymbol{z})=h\left(y-\gamma_{0}^{T} \boldsymbol{z}\right),
$$

where $h(\cdot)$ is an unknown distribution function. The estimator $\hat{\gamma}$ of $\gamma_{0}$ can be obtained by solving

$$
\sum_{i=1}^{n}\left(Y_{i}-\gamma^{T} \boldsymbol{Z}_{i}\right) \boldsymbol{Z}_{i}=0
$$

The function $h(y)$ can be estimated by

$$
\hat{h}(y ; \hat{\gamma})=n^{-1} \sum_{i=1}^{n} I\left(Y_{i}-\hat{\gamma}^{T} \boldsymbol{Z}_{i} \leq y\right) .
$$

Let $\tilde{\beta}$ be the solution to the partial likelihood estimating equation with $F_{Y \mid \boldsymbol{Z}}(y \mid \boldsymbol{z})$ replaced by $\hat{h}\left(y-\hat{\gamma}^{T} \boldsymbol{z}, \hat{\gamma}\right)$ and $\tilde{\Lambda}_{0}(t)$ be the corresponding Breslow estimator of $\Lambda_{0}(t)$. An estimator of $\theta(v \mid \boldsymbol{z})$ is

$$
\tilde{\theta}^{*}(v \mid \boldsymbol{Z})=\int_{0}^{L} \int_{v}^{1} \tilde{S}(t \mid v, \boldsymbol{z}, 1) d u d t+\int_{0}^{L} \int_{0}^{v} \tilde{S}(t \mid v, \boldsymbol{z}, 0) d u d t
$$

where

$$
\tilde{S}(t \mid v, \boldsymbol{z}, a)=\exp \left\{-\tilde{\Lambda}_{0}(t) \exp \left(\tilde{\beta}^{T} g(v, \boldsymbol{z}, a)\right)\right\} .
$$

Note that both $\tilde{\theta}$ and $\tilde{\theta}^{*}$ can also be used in the case of no covariates. Since these estimators are semiparametric estimators, they are more efficient than the nonparametric estimator 
$\hat{\theta}$ under the correct model assumptions. However, if the model assumptions are violated, inference based on these estimators can be misleading. We may need to transform the biomarker appropriately to assure that the proportional hazards assumption holds. In contrast, the nonparametric approach is invariant to monotone increasing transformations.

Under some regularity conditions, we can show that both $n^{1 / 2}\{\tilde{\theta}(v \mid \boldsymbol{z})-\theta(v \mid \boldsymbol{z})\}$ and $n^{1 / 2}\left\{\tilde{\theta}^{*}(v \mid \boldsymbol{z})-\theta(v \mid \boldsymbol{z})\right\}$ converge to zero-mean Gaussian processes. The derivation is involved with application of empirical process and U-process theories. The details of the asymptotic distribution and the proof are given in Appendix B. A consistent estimator for the variance of $\tilde{\theta}(v \mid \boldsymbol{z})$ is given in equation (B.4), which involves complex integrals. The variance formula for $\tilde{\theta}^{*}(v)$ is even more complicated and involves the derivative of the unknown function $h(\cdot)$. In practice, by analogy to the nonparametric case, we can use the bootstrap method to compute estimates of the standard errors and confidence bands for $\tilde{\theta}(v \mid \boldsymbol{z})$ and $\tilde{\theta}^{*}(v \mid \boldsymbol{z})$.

\section{SIMULATION STUDIES}

Simulation studies were conducted to assess the finite sample properties of the estimators. We first considered the simple case of no covariates when both the nonparametric and semiparametric estimators can be used. We generated data for 300 and 600 patients in a randomized trial with $\mathrm{P}[A=1]=0.5$. The marker $Y$ was generated from the standard normal distribution. The survival time was generated according to the proportional hazards model (6) with $g(v, \boldsymbol{z}, a)=(v, a, v a)^{T}, \beta=(-4,-2,1)^{T}$, and $\lambda_{0}(t)=0.1$. Censoring time was generated from an exponential distribution with mean 30 and truncated at 20, leading to a censoring rate of about 30\%. Consider $L=15$, and the SI curve is shown in Fig. 4.

We estimated the SI curve using $\hat{\theta}, \tilde{\theta}$ and $\tilde{\theta}^{*}$ for 500 simulated data sets. The estimated standard errors were computed by the bootstrap method using 100 resampled data sets for all the estimators. For each estimator, the $95 \%$ Wald confidence intervals were calculated. Table 1 presents the results for $v=0.1, \ldots, 0.9$. All the estimators exhibit negligible bias, and the standard errors track the true standard deviations of the estimators well. The coverage probabilities are close to their nominal levels. As expected, $\tilde{\theta}^{*}$ is less efficient than $\tilde{\theta}$ because the true $F_{Y}$ is used for the former while $F_{Y}$ is estimated for the latter, but the efficiency loss is small. Moreover, $\tilde{\theta}^{*}$ is more efficient than the nonparametric estimator $\hat{\theta}$ as expected.

To investigate the robustness of the estimators, we also conducted simulations under similar scenarios as above except that the survival time was generated from a gamma distribution with the shape parameter equal to 4 and the scale parameter equal to $\left\{5 \lambda_{i}\left(t, Y_{i}, \boldsymbol{Z}_{i}\right)\right\}^{-1}$, where $\lambda_{i}\left(t, Y_{i}, \boldsymbol{Z}_{i}\right)$ is the hazard in the simulation above. In this case, the survival model is misspecified for the semiparametric estimators. The results are shown in Table 2. The nonparametric estimator $\hat{\theta}$ still works well while the semiparametric estimators are obviously biased with poor coverage probabilities for the confidence intervals.

We then considered the case when a covariate $\boldsymbol{Z}$ was included. The covariate $\boldsymbol{Z}$ was generated from a standard normal distribution. The biomarker $Y$ equals $\boldsymbol{Z}$ plus a standard normal error. The survival time followed the proportional hazards model (6) with $g(v, \boldsymbol{z}, a)=(v, a, z, v a, v z, a z, v a z)^{T}, \beta=(1,-0.7,-0.1,-0.9,-0.3,0.8,-0.9)$, and $\lambda_{0}(t)=0.2$. Censoring time was generated from an exponential distribution with mean 20 and truncated at 8 , leading to a censoring rate of about $30 \%$. Consider $L=5$ and the 
covariate SI curves for $z=-1,0,1$ are shown in Fig. 5. For 500 simulated datasets with $n=200$ and 400, We estimated the covariate specific SI curves for 500 simulated datasets with $n=200$ and 400 using $\tilde{\theta}$ and $\tilde{\theta}^{*}$. The results in Table 3 show similar patterns as above and both estimators work well.

In summary, the nonparametric estimator $\hat{\theta}$ is robust while the semiparametric estimators $\tilde{\theta}^{*}$ and $\hat{\theta}$ depend on the correct specification of the models. Obvious deviances between these estimators may indicate violation of the model assumptions for the semiparametric estimators. We recommend using the nonparametric estimator when there is no covariates. Extension of the semiparametric estimators to more flexible models is discussed in Section 6.

\section{APPLICATION}

As an illustration, we applied the proposed approaches to a subset of the ECOG clinical trial, which was analyzed by Li and Ryan (2006). In this subset, disease progression free survival and c-myc expression level was measured on 92 patients randomized to receive surgery alone or surgery plus chemotherapy, among which 47 were males and 45 were females. We are interested in assessing whether the c-myc expression level can be used to select patients for surgery alone versus surgery plus chemotherapy. We estimated the SI curve using both the nonparametric and semiparametric methods. For the semiparametric approach, we included $\log$ c-myc expression level $Y$, treatment indicator $A=I$ (surgery plus chemotherapy) and their interaction in the the proportional hazards model. This model was also used in Li and Ryan (2006). This corresponds to $g(v, \boldsymbol{z}, a)=(v, a, v a)^{T}$ in the proportional hazards model (6). We consider the mean disease progression free survival time restricted to five years. Since the distribution $F_{Y}(y)$ is unknown, we estimated the SI curve using the estimators $\hat{\theta}(v)$ and $\tilde{\theta}^{*}(v)$. The estimates and the $95 \%$ pointwise confidence intervals and simultaneous confidence bands are shown in the left panel of Figure 6. The semiparametric estimate is essentially a smoothed version of the nonparametric estimate, which indicates that the corresponding model assumptions are appropriate for this dataset. The estimated SI curves seem to decrease with $v$. That is, assigning all patients to surgery plus chemotherapy may achieve the maximum mean survival time within 5 years. However, this may not be the optimal treatment policy considering that surgery plus chemotherapy is more invasive than surgery alone and the estimated SI curve is almost horizonal for $v$ in $(0,0.3)$.

To take into account of the side effect of chemotherapy, we may consider a utility measure $U$, for example, $U=T^{*}-d A$, where $d$ is a nonnegative weight denoting the deteriorating effect of chemotherapy on the survival time; that is, a person may prefer surgery alone if the additional chemotherapy does not lengthen the survival time by at least $d$. The SI curve based on $U$ can be obtained by shifting the SI curve based on $T^{*}$ by $w(1-v)$ and hence can be easily estimated. The optimal threshold can be achieved by maximizing the SI curve based on $U$. The right panel of Figure 6 shows the corresponding nonparametric and semiparametric estimates with $d=0.5$. The maximum point is achieved at $v=0.32$ for the semiparametric SI curve estimate. This indicates that, considering the c-myc expression level and the side effect of chemotherapy, the optimal treatment policy may be the one that assigns patients whose c-myc expression level falls below the $32 \%$ percentile to surgery alone. The maximum point of the nonparametric estimate may be less stable since the non-smoothness of the curve. We do not see significant difference on either estimate across $v$ which may due to the small sample size of this dataset. Whether the difference is clinically important may be worth future investigation with larger clinical trials. 
As we have discussed in Section 1, it is also of interest to assess whether the gender of the patient may affect the treatment selection. Although we may estimate a separate SI curve for males and females, the estimate may be unreliable because of the small sample size in each group. We thus estimated the covariate specific SI curve based on $T$ adjusting for $Z=I$ (gender=male) using the semiparametric estimator $\tilde{\theta}^{*}(v \mid \boldsymbol{z})$ by further including $Z$ and all the two-way and three-way interactions in the proportional hazards model considered above. The left panel of Figure 7 shows the results. For male patients, the SI curve seems to be horizontal before $v=0.3$ and decline thereafter, while for female patients the SI curve tends to decrease for $v$ from 0 to 1 . This indicates the optimal thresholds treatment selection should be different for males and females. To obtain the optimal thresholds, the covariate specific SI curves based on $U$ were also estimated as shown in the right panel of Figure 7. The estimated optimal threshold is $v=0.25$ for males and $v=0.05$ for females. Thus we may want to assign almost all females to surgery plus chemotherapy while assign male patients whose c-myc expression level fall below the $25 \%$ percentile to surgery alone.

\section{DISCUSSION}

We have proposed the SI curve for the survival time to evaluate the impact of a treatment selection policy based on $Y>y_{v}$. Both nonparametric and semiparametric estimators are derived. We recommend using the nonparametric estimator when it is not necessary to adjust for covariates because of its robustness. The semiparametric estimators are less robust, but can be more efficient under the correctly specified model. In addition, they can easily incorporate covariates in estimation. Whether to use the unadjusted or adjusted SI curve depends on the specific treatment policy, that is, whether we would like to select the treatment based on the biomarker alone.

For the semiparametric approach, we have used the standard proportional hazards model to characterize the relationship between the survival time and the marker and the covariates. This can be easily adapted to more flexible models. For example, we can use other survival models, such as the stratified proportional hazards model, the accelerated failure time model and the transformation model, as long as we can obtain consistent estimators for the survival distribution. The nonparametric transformation model (Song et al., 2007) may be an attractive extension as it includes most popular survival models as special cases, such as the proportional hazards model and the accelerated failure time model. The semiparametric location model (7) is used only for estimating the conditional distribution function $F_{Y \mid Z}$. We can remove this assumption and estimate $F_{Y \mid Z}$ by kernel smoothing method when the number of covariates is small and the sample size is relatively large, since the kernel smoothing method may not work well otherwise.

Although the SI curve is proposed based on the restricted mean survival time, it can be extended to other statistical measures. For example, a utility function that incorporates notions of cost and quality of life might be employed. Although we have focused on survival time as outcomes, the approach can be adapted to discrete and continuous outcomes with minor modifications.

In this paper, we consider SI curves based on a single biomarker. In practice, there may exist multiple biomarkers. For example, multiple genes may be related to a specific disease or affect the survival time. An important issue is how to combine these biomarkers for treatment selection. On the other hand, it may be needed to select among more than two treatments. These issues will be investigated in our future research. 


\section{ACKNOWLEDGEMENTS}

This work is supported in part by the UGARF grant from the University of Georgia (Song) and the VA Clinical R\&D funding (Zhou and Song). Zhou's work is supported in part by AHRQ grant R01HS013105 and VA HSR\&D grant ECI-03-206. This paper presents the findings and conclusions of the authors. It does not necessarily represent those of VA HSR\&D Service. We would like to thank Dr. Yi Li and ECOG for providing the cancer data.

\section{Appendix A. ASYMPTOTIC PROPERTIES OF $\theta(v)$}

Now we derive the asymptotic properties of $\hat{\theta}(v)$ based primarily on empirical process theory. Let $\mathcal{Y}$ be the support of $Y$ and $F_{Y}(y)=P(Y \leq y)$ be the distribution function of $Y$. We assume the following regularity conditions:

A. $T$ and $C$ are independent given $Y$ and $A$;

B. $P\left(X \geq L, A=1, Y>y_{q}\right)>0$ and $P\left(X \geq L, A=0, Y<y_{p}\right)>0$ for some constants $p$ and $q, 0<p<q<1$

C. For $y \in \mathcal{Y}$ and $a \in\{0,1\}, S^{(a)}(u, y)$ is an absolutely continuous distribution function of $u$ for $u \in[0,+\infty)$;

D. $F_{Y}(y)$ is continuously differentiable with a positive derivative $f_{Y}(y)$ on the interval $\left[F^{(-1)}(p)-\varepsilon, F^{(-1)}(q)+\varepsilon\right]$ for some $\varepsilon>0$.

Let $\hat{E}$ be the empirical average operator such that $\hat{E} c=n^{-1} \sum_{i=1}^{n} c_{i}$. Let

$$
\hat{\Lambda}^{(a)}(t, y)=\int_{0}^{t} \frac{\hat{E} N^{(a)}(d s, y)}{\hat{E} R^{(a)}(s, y)}
$$

be the Nelson-Aalen estimator of $\Lambda^{(a)}(t, y), a=0,1$. Using the functional delta method, we can show the following lemma:

Lemma 1. $n^{1 / 2}\left\{\hat{\Lambda}^{(a)}(t, y)-\Lambda^{(a)}(t, y)\right\}$ converges to a Gaussian process $\mathbb{H}^{(a)}(t, y)$ in $[0, L] \times$ $\left[y_{p}, y_{q}\right]$ with mean zero and covariance

$$
\operatorname{cov}\left\{\mathbb{H}^{(a)}\left(t_{1}, y_{1}\right), \mathbb{H}^{(a)}\left(t_{2}, y_{2}\right)\right\}=\operatorname{cov}\left\{\omega_{i}^{(a)}\left(t_{1}, y_{1}\right), \omega_{i}^{(a)}\left(t_{2}, y_{2}\right)\right\}
$$

where

$$
\omega_{i}^{(a)}(t, y)=\int_{0}^{t}\left[\frac{N_{i}^{(a)}(d u, y)}{E R^{(a)}(u, y)}-\frac{E N^{(a)}(d u, y) R_{2 i}^{(a)}(u, y)}{\left\{E R^{(a)}(u, y)\right\}^{2}}\right]
$$

Then, under conditions A-C, applying the functional delta method to the product integral (van der Vaart and Wellner 2000, chap. 3.9.4.5), we can show the following lemma:

Lemma 2. $n^{1 / 2}\left\{\hat{S}^{(a)}(t, y)-S^{(a)}(t, y)\right\}$ converges to the Gaussian process $-S^{(a)}(t, y) \mathbb{H}^{(a)}(t, y)$ in $[0, L] \times\left[y_{p}, y_{q}\right]$. 
Using empirical process theory, we know that the empirical process $n^{1 / 2}\left\{\hat{F}_{Y}-F_{Y}\right\}$ converges to a Gaussian process $\mathbb{F}$. Since $\hat{\theta}(v)$ is a composite function of $\hat{S}^{(0)}, \hat{S}^{(1)}$ and $\hat{F}_{Y}$, the asymptotic distribution of $\hat{\theta}(v)$ is then derived using Lemma 2 and condition D and using the functional delta method (van der Vaart and Wellner 2000, chap. 3.9) with some simple algebra.

Theorem 1. $n^{1 / 2}\{\hat{\theta}(v)-\theta(v)\}$ converges on the interval $[p, q]$ to a Gaussian process

$$
\begin{aligned}
\mathbb{G}(v)= & -(1-v) \int_{0}^{L}\left[S^{(1)}\left(t, y_{v}\right) \mathbb{H}^{(1)}\left(t, y_{v}\right)\right. \\
& \left.+S^{(1)}\left(t, y_{v}\right) \frac{\partial \Lambda^{(1)}\left(t, y_{v}\right)}{\partial y}\left\{1-\Lambda^{(1)}\left(t, y_{v}\right)\right\} \frac{\mathbb{F}\left(y_{v}\right)}{f_{Y}\left\{F_{Y}^{-1}(v)\right\}}\right] d t \\
& -v \int_{0}^{L}\left[S^{(0)}\left(t, y_{v}\right) \mathbb{H}^{(0)}\left(t, y_{v}\right)\right. \\
& \left.+S^{(0)}\left(t, y_{v}\right) \frac{\partial \Lambda^{(0)}\left(t, y_{v}\right)}{\partial y}\left\{1-\Lambda^{(0)}\left(t, y_{v}\right)\right\} \frac{\mathbb{F}\left(y_{v}\right)}{f_{Y}\left\{F_{Y}^{-1}(v)\right\}}\right] d t
\end{aligned}
$$

with mean zero and covariance $\operatorname{cov}\left\{\mathbb{G}\left(v_{1}\right), \mathbb{G}\left(v_{2}\right)\right\}=\operatorname{cov}\left\{\sigma\left(v_{1}\right), \sigma\left(v_{2}\right)\right\}$, where

$$
\begin{aligned}
\sigma(v)= & -(1-v) \int_{0}^{L}\left[S^{(1)}\left(t, y_{v}\right) \omega_{i}^{(1)}\left(t, y_{v}\right)\right. \\
& \left.+S^{(1)}\left(t, y_{v}\right) \frac{\partial \Lambda^{(1)}\left(t, y_{v}\right)}{\partial y}\left\{1-\Lambda^{(1)}\left(t, y_{v}\right)\right\} \frac{I\left(Y_{i} \leq y_{v}\right)}{f_{Y}\left\{F_{Y}^{-1}(v)\right\}}\right] d t \\
& -v \int_{0}^{L}\left[S^{(0)}\left(t, y_{v}\right) \omega_{i}^{(0)}\left(t, y_{v}\right)\right. \\
& \left.+S^{(0)}\left(t, y_{v}\right) \frac{\partial \Lambda^{(0)}\left(t, y_{v}\right)}{\partial y}\left\{1-\Lambda^{(0)}\left(t, y_{v}\right)\right\} \frac{I\left(Y_{i} \leq y_{v}\right)}{f_{Y}\left\{F_{Y}^{-1}(v)\right\}}\right] d t .
\end{aligned}
$$

\section{Appendix B. ASYMPTOTIC PROPERTIES OF $\tilde{\theta}(v \mid \boldsymbol{z})$ AND $\tilde{\theta}^{*}(v \mid \boldsymbol{z})$}

Regularity Conditions. Let $\mathcal{Z}$ be the support of $\boldsymbol{Z}, \tau$ be a constant such that $\tau \geq L$, $W_{i}=\left(V_{i}, A_{i}, X_{i}, \Delta_{i}\right)$, and

$$
Q_{b i}(\beta ; W, s)=R_{i}(s) g^{\otimes b}\left(V_{i}, \boldsymbol{Z}_{i}, A_{i}\right) \exp \left\{\beta^{T} g\left(V_{i}, \boldsymbol{Z}_{i}, A_{i}\right)\right\} \text {, for } b=0,1,2,
$$

where for any vector $c, c^{\otimes r}=0,1, c c^{T}$ for $r=0,1,2$, respectively. To derive the asymptotic properties for $\tilde{\theta}(v \mid \boldsymbol{z})$, we assume the following regularity conditions:

$\mathrm{A}^{\prime} . T$ and $C$ are independent given $Y, A$ and $\boldsymbol{Z}$;

$B^{\prime} . P(X \geq L) \geq P(X \geq \tau)>0$;

$\mathrm{C}^{\prime}$. For $v \in(0,1), \boldsymbol{z} \in \mathcal{Z}$ and $a=0,1, S(t \mid v, \boldsymbol{z}, a)$ is an absolutely continuous function of $t$ for $t \in[0,+\infty)$; 
$\mathrm{D}^{\prime} . \Gamma\left(\beta_{0}\right)=\int_{0}^{\tau}\left\{\frac{E Q_{2}\left(\beta_{0} ; W, s\right)}{E Q_{0}\left(\beta_{0} ; W, s\right)}-\frac{E Q_{1}^{2}\left(\beta_{0} ; W, s\right)}{E Q_{0}^{2}\left(\beta_{0} ; W, s\right)}\right\} E d N(s)$ is positive definite;

$\mathrm{E}^{\prime}$. For $v \in(0,1), \boldsymbol{z} \in \mathcal{Z}$ and $a=0,1, g(v, \boldsymbol{z}, a)$ is bounded and has uniformly continuous and bounded partial derivative $\dot{g}(v, \boldsymbol{z}, a)=\partial g(v, \boldsymbol{z}, a) / \partial v$;

$\mathrm{F}^{\prime} . E\left(\boldsymbol{Z}^{T} \boldsymbol{Z}\right)<\infty, E\left(Y^{2}\right)<\infty, E\left(\boldsymbol{Z} \boldsymbol{Z}^{T}\right)$ is positive definite;

$\mathrm{G}^{\prime} . h(y)$ is bounded and has first- and second- order bounded derivatives $h^{\prime}(y)$ and $h^{\prime \prime}(y)$ for $y \in(-\infty,+\infty)$.

The asymptotic properties for $\tilde{\theta}(v \mid \boldsymbol{z})$ and $\tilde{\theta}^{*}(v \mid \boldsymbol{z})$ are summarized in the following theorems.

Theorem 2. Given $\boldsymbol{z} \in \mathcal{Z}, n^{1 / 2}\{\tilde{\theta}(v \mid \boldsymbol{z})-\theta(v \mid \boldsymbol{z})\}$ converges to a zero-mean Gaussian process $\mathbb{G}^{*}(v, \boldsymbol{z})$ with the covariance given in Appendix $B$.

Theorem 3. Given $\boldsymbol{z} \in \mathcal{Z}, n^{1 / 2}\left\{\tilde{\theta}^{*}(v \mid \boldsymbol{z})-\theta(v \mid \boldsymbol{z})\right\}$ converges to a zero-mean Gaussian process $\mathbb{G}^{\#}(v, \boldsymbol{z})$ on the interval $[p, q]$ with the covariance given in Appendix $B$.

Derivation of Asymptotic Properties of $\tilde{\theta}(v \mid \boldsymbol{z})$. Under conditions $\mathrm{A}^{\prime}-\mathrm{E}^{\prime}$, the asymptotic properties of $\hat{\beta}, \hat{\Lambda}_{0}(t)$ and $\hat{S}(t \mid v, \boldsymbol{z}, a)$ have been shown in Andersen and Gill (1982). Since $\theta(v \mid \boldsymbol{z})$ is a composite functional differentiable with respect to $S(t \mid v, \boldsymbol{z}, a), \Lambda_{0}(t)$ and $\beta_{0}$, using the functional delta method, $n^{1 / 2}\{\tilde{\theta}(v \mid \boldsymbol{z})-\theta(v \mid \boldsymbol{z})\}$ converges to a Gaussian process $\mathbb{G}_{\boldsymbol{z}}^{*}(v, \boldsymbol{z})$ for $v \in(p, q)$ with mean zero. Next we derive the covariance of $\mathbb{G}_{\boldsymbol{z}}^{*}(v, \boldsymbol{z})$.

The partial likelihood estimating equation can be written as

$$
\hat{U}(\beta ; W)=\int_{0}^{\tau}\left\{\hat{E} g(V, \boldsymbol{Z}, A) d N(s)-\frac{\hat{E} Q_{1}\left(\beta ; W_{i}, s\right)}{\hat{E} Q_{0}\left(\beta ; W_{i}, s\right)} \hat{E} d N(s)\right\}=0,
$$

Let $\hat{\Gamma}(\beta)=\frac{\partial \hat{U}(\beta)}{\partial \beta}$. Using arguments similar to those in the appendix of Huang and Wang (2000), we can show that

$$
n^{1 / 2}\left\{\hat{\beta}-\beta_{0}\right\}=n^{-1 / 2} \sum_{i=1}^{n} \pi\left(\beta_{0} ; W_{i}\right)+o_{p}(1)
$$

where

$$
\begin{aligned}
& \pi\left(\beta ; W_{i}\right)=\Gamma^{-1}(\beta) \rho\left(\beta ; W_{i}\right), \text { and } \\
& \rho\left(\beta ; W_{i}\right)=\int_{0}^{\tau}\left\{g\left(V_{i}, \boldsymbol{Z}_{i}, A_{i}\right)-\frac{E Q_{1}\left(\beta_{0} ; W_{i}, s\right)}{E Q_{0}\left(\beta_{0} ; W_{i}, s\right)}\right\}\left\{d N_{i}(s)-\frac{Q_{0 i}\left(\beta_{0} ; W_{i}, s\right)}{E Q_{0}\left(\beta_{0} ; W_{i}, s\right)} E d N(s)\right\} .
\end{aligned}
$$

The Breslow estimator for the cumulated baseline hazard can be written as

$$
\hat{\Lambda}_{0}(t)=\int_{0}^{t} \frac{\hat{E} d N(s)}{\hat{E} Q_{0}\left(\beta ; W_{i}, s\right)} .
$$

Using the functional delta method, we can show that $n^{1 / 2}\{\hat{S}(t \mid u, \boldsymbol{z}, a)-S(t \mid u, \boldsymbol{z}, a)\}$ converges to a Gaussian process with mean zero. With the functional Taylor expansion, 
straightforward algebra leads to

$$
\begin{aligned}
n^{1 / 2}\left\{\hat{\Lambda}_{0}(t)-\Lambda_{0}(t)\right\}= & n^{-1 / 2} \sum_{i=1}^{n} \delta\left(t, \beta_{0}, W_{i}\right) \\
& -\int_{0}^{t} \frac{E Q_{1}^{T}\left(\beta_{0} ; W_{i}, s\right) E d N(s)}{E^{2} Q_{0}\left(\beta_{0} ; W_{i}, s\right)} n^{1 / 2}\left\{\hat{\beta}-\beta_{0}\right\}+o_{p}(1),(
\end{aligned}
$$

and

$$
\begin{aligned}
& n^{1 / 2}\{\hat{S}(t \mid u, \boldsymbol{z}, a)-S(t \mid u, \boldsymbol{z}, a)\} \\
= & -S(t \mid u, \boldsymbol{z}, a) \exp \left\{\beta_{0}^{T} g(u, \boldsymbol{z}, a)\right\} n^{1 / 2}\left\{\hat{\Lambda}_{0}(t)-\Lambda_{0}(t)\right\} \\
& -S(t \mid u, \boldsymbol{z}, a) \exp \left\{\beta_{0}^{T} g(u, \boldsymbol{z}, a)\right\} \Lambda_{0}(t) g^{T}(u, \boldsymbol{z}, a) n^{1 / 2}\left\{\hat{\beta}-\beta_{0}\right\} \\
& +o_{p}(1),
\end{aligned}
$$

where

$$
\delta\left(t, \beta, W_{i}\right)=\int_{0}^{t} \frac{d N_{i}(s) E Q_{0}\left(\beta ; W_{i}, s\right)-R_{i}(s) \exp \left\{\beta^{T} g\left(V_{i}, \boldsymbol{Z}_{i}, A_{i}\right)\right\} E d N(s)}{E^{2} Q_{0}\left(\beta ; W_{i}, s\right)} .
$$

Substituting (B.1) and (B.2) in (B.3), we have

$$
n^{1 / 2}\{\hat{S}(t \mid u, \boldsymbol{z}, a)-S(t \mid u, \boldsymbol{z}, a)\}=n^{-1 / 2} \sum_{i=1}^{n} \xi\left(\beta_{0}, t, u, \boldsymbol{z}, a, W_{i}\right)+o_{p}(1)
$$

where

$$
\begin{aligned}
& \xi\left(\beta, t, u, \boldsymbol{z}, a, W_{i}\right)=-S(t \mid u, \boldsymbol{z}, a) \exp \left\{\beta^{T} g(u, \boldsymbol{z}, a)\right\} \\
& \quad \times\left(\delta\left(t, \beta, W_{i}\right)-\left\{\int_{0}^{t} \frac{E Q_{1}^{T}\left(\beta ; W_{i}, s\right) E d N(s)}{E^{2} Q_{0}\left(\beta ; W_{i}, s\right)}-\Lambda_{0}(t) g^{T}(u, \boldsymbol{z}, a)\right\} \pi\left(\beta ; W_{i}\right)\right) .
\end{aligned}
$$

Therefore

$$
\begin{aligned}
n^{1 / 2}\{\hat{\theta}(v \mid \boldsymbol{z})-\theta(v \mid \boldsymbol{z})\}= & \sum_{i=1}^{n} \int_{v}^{1} \int_{0}^{L} n^{1 / 2}\{\hat{S}(t \mid u, \boldsymbol{z}, 1)-S(t \mid u, \boldsymbol{z}, a)\} d t d u \\
& +\int_{0}^{v} \int_{0}^{L} n^{1 / 2}\{\hat{S}(t \mid u, \boldsymbol{z}, 1)-S(t \mid u, \boldsymbol{z}, a)\} d t d u+o_{p}(1) \\
= & n^{1 / 2} \sum_{i=1}^{n} \mu_{i}(v ; \boldsymbol{z})+o_{p}(1),
\end{aligned}
$$

where

$$
\mu_{i}(v ; \boldsymbol{z})=\int_{v}^{1} \int_{0}^{L} \xi\left(\beta, t, u, \boldsymbol{z}, 1, X_{i}, \boldsymbol{Z}_{i}, A_{i}\right) d t d u+\int_{0}^{v} \int_{0}^{L} \xi\left(\beta, t, u, \boldsymbol{z}, 0, X_{i}, \boldsymbol{Z}_{i}, A_{i}\right) d t d u .
$$

Hence $\operatorname{cov}\left\{\mathbb{G}^{*}\left(v_{1} ; \boldsymbol{z}\right), \mathbb{G}^{*}\left(v_{2} ; \boldsymbol{z}\right)\right\}=\operatorname{cov}\left\{\mu\left(v_{1} ; \boldsymbol{z}\right), \mu\left(v_{2} ; \boldsymbol{z}\right)\right\}=\Sigma\left(v_{1}, v_{2} ; \boldsymbol{z}\right)$ for $v_{1}, v_{2} \in(p, q)$. An estimator of the variance $\operatorname{var}\{\mu(v ; \boldsymbol{z})\}=\Sigma(v, v ; \boldsymbol{z})$ is

$$
\hat{\Sigma}(v, v ; \boldsymbol{z})=n^{-1} \sum_{i=1}^{n}\left\{\hat{\mu}_{i}(v ; \boldsymbol{z})-\overline{\hat{\mu}}(v ; \boldsymbol{z})\right\}^{2}
$$


where $\overline{\hat{\mu}}(v ; \boldsymbol{z})=n^{-1} \sum_{i=1}^{n} \hat{\mu}_{i}(v ; \boldsymbol{z})$,

$$
\begin{aligned}
\hat{\mu}_{i}(v ; \boldsymbol{z})= & \int_{v}^{1} \int_{0}^{L} \hat{\xi}\left(\hat{\beta}, t, u, \boldsymbol{z}, 1, X_{i}, \boldsymbol{Z}_{i}, A_{i}\right) d t d u+\int_{0}^{v} \int_{0}^{L} \hat{\xi}\left(\hat{\beta}, t, u, \boldsymbol{z}, 0, X_{i}, \boldsymbol{Z}_{i}, A_{i}\right) d t d u, \\
\hat{\xi}\left(\beta, t, u, \boldsymbol{z}, 1, X_{i}, \boldsymbol{Z}_{i}, A_{i}\right)=-\hat{S}(t \mid u, \boldsymbol{z}, a) \exp \left\{\beta^{T} g(u, \boldsymbol{z}, a)\right\} & \\
& \times\left(\hat{\delta}\left(t, \beta, W_{i}\right)-\left\{\int_{0}^{t} \frac{\hat{E} Q_{1}^{T}\left(\beta ; W_{i}, s\right) \hat{E} d N(s)}{\hat{E}^{2} Q_{0}\left(\beta ; W_{i}, s\right)}-\hat{\Lambda}_{0}(t) g^{T}(u, \boldsymbol{z}, a)\right\} \hat{\pi}\left(\beta_{0} ; W_{i}\right)\right), \\
\hat{\delta}\left(t, \beta, W_{i}\right)= & \int_{0}^{t} \frac{d N_{i}(s) \hat{E} Q_{0}\left(\beta ; W_{i}, s\right)-R_{i}(s) \exp \left\{\beta^{T} g\left(V_{i}, \boldsymbol{Z}_{i}, A_{i}\right)\right\} \hat{E} d N(s)}{\hat{E}^{2} Q_{0}\left(\beta ; W_{i}, s\right)} \\
\hat{\pi}\left(\beta ; W_{i}\right)= & \hat{\Gamma}^{-1}(\beta) \hat{\rho}\left(\beta ; W_{i}\right), \\
\hat{\rho}\left(\beta ; W_{i}\right)= & \int_{0}^{\tau}\left\{g\left(V_{i}, \boldsymbol{Z}_{i}, A_{i}\right)-\frac{\hat{E} Q_{1}\left(\beta_{0} ; W_{i}, s\right)}{\hat{E} Q_{0}\left(\beta_{0} ; W_{i}, s\right)}\right\}\left\{d N_{i}(s)-\frac{Q_{0 i}\left(\beta_{0} ; W_{i}, s\right)}{\hat{E} Q_{0}\left(\beta_{0} ; W_{i}, s\right)} \hat{E} d N(s)\right\}, \\
\hat{\Gamma}(\beta)= & \int_{0}^{\tau}\left\{\frac{\hat{E} Q_{2}(\beta ; W, s)}{\hat{E} Q_{0}(\beta ; W, s)}-\frac{\hat{E} Q_{1}^{2}(\beta ; W, s)}{\hat{E} Q_{0}^{2}(\beta ; W, s)}\right\} \hat{E} d N(s) .
\end{aligned}
$$

Derivation of Asymptotic Properties of $\tilde{\theta}^{*}(v \mid \boldsymbol{z})$. Now, we consider the asymptotic properties of $\tilde{\theta}^{*}(v \mid \boldsymbol{z})$. We only need to show that $n^{1 / 2}\left(\tilde{\beta}-\beta_{0}\right)$ is asymptotically normal and then use similar proofs as those for $\tilde{\theta}(v \mid \boldsymbol{z})$. Let $\hat{V}_{i}=\hat{h}\left(Y_{i} ; \hat{\gamma}\right)$, and $\hat{W}_{i}=\left(\hat{V}_{i}, A_{i}, X_{i}, \Delta_{i}\right)$. First, under condition $\mathrm{F}^{\prime}$, by the Slutsky theorem,

$$
n^{1 / 2}\left(\hat{\gamma}-\gamma_{0}\right)=\left(n^{-1} \sum_{i=1}^{n} \boldsymbol{Z}_{i} \boldsymbol{Z}_{i}^{T}\right)^{-1} n^{-1 / 2} \sum_{i=1}^{n} \boldsymbol{Z}_{i}\left(Y_{i}-\gamma_{0} \boldsymbol{Z}_{i}^{T}\right)
$$

converges in distribution to a zero-mean normal random variable. Let $\mathcal{N}\left(\gamma_{0}\right)$ be a compact neighborhood of $\gamma_{0}$. By the functional central limit theorem,

$$
n^{1 / 2}\left[\hat{h}\left(y-\gamma^{T} z, \gamma\right)-E h\left\{y+\gamma^{T}(Z-z)-\gamma_{0} Z\right\}\right]
$$

converges to a mean zero Gaussian process on $(y, \gamma) \in \mathcal{Y} \times \mathcal{N}\left(\gamma_{0}\right)$. It follows from the equicontinuity of the foregoing process and the consistency of $\hat{\gamma}$ that

$$
\begin{array}{r}
\sup _{y \in \mathcal{Y}} \mid n^{1 / 2}\left[\hat{h}\left(y-\hat{\gamma}^{T} z, \hat{\gamma}\right)-E h\left\{y+\hat{\gamma}^{T}(Z-z)-\gamma_{0} Z\right\}\right] \\
-n^{1 / 2}\left[\hat{h}\left(y-\gamma_{0}^{T} z, \hat{\gamma}\right)-h\left\{y-\gamma_{0}^{T} Z\right\}\right] \mid=o_{p}(1) .
\end{array}
$$

This implies that

$$
\begin{gathered}
n^{1 / 2}\left\{\hat{h}\left(y-\hat{\gamma}^{T} z, \hat{\gamma}\right)-h\left(y-\gamma_{0}^{T} z\right)\right\}=n^{1 / 2}\left\{\hat{h}\left(y-\gamma_{0}^{T} z, \hat{\gamma}\right)-h\left(y-\gamma_{0}^{T} z\right)\right\} \\
+n^{1 / 2}\left\{E h\left\{y+\hat{\gamma}^{T}(Z-z)-\gamma_{0} Z\right\}-h\left(y-\gamma_{0}^{T} z\right)\right\}+o_{p}(1)
\end{gathered}
$$

uniformly for $y \in \mathcal{Y}$. By a Taylor series expansion, under condition $\mathrm{G}^{\prime}$, the second term in (B.6) can be written as

$$
h^{\prime}(y)\left\{E^{T}(Z)-z\right\} n^{-1 / 2}\left(\hat{\gamma}-\gamma_{0}\right)+o_{p}(1)
$$


uniformly for $y \in \mathcal{Y}$. This, together with (B.5), implies that

$$
\begin{aligned}
n^{1 / 2}\{ & \left.\hat{h}\left(y-\hat{\gamma}^{T} z, \hat{\gamma}\right)-h\left(y-\gamma_{0}^{T} z\right)\right\} \\
= & n^{-1 / 2} \sum_{i=1}^{n}\left[\left\{I\left(Y_{i}-\gamma_{0}^{T} Z_{i} \leq y-\gamma_{0}^{T} z\right)-h\left(y-\gamma_{0}^{T} z\right)\right\}\right. \\
\quad & \left.\quad h^{\prime}\left(y-\gamma_{0}^{T} z\right)\{E(Z)-z\}^{T}\left\{E\left(Z Z^{T}\right)\right\}^{-1} Z_{i}\left(Y_{i}-Z_{i}^{T} \gamma_{0}\right)\right]+o_{p}(1)
\end{aligned}
$$

uniformly for $y \in \mathcal{Y}$. With arguments similar to those in Song and Pepe (2004), under condition $\mathrm{E}^{\prime}$,

$$
\begin{aligned}
n^{1 / 2} & \{\hat{U}(\beta ; \hat{W})-\hat{U}(\beta ; W)\} \\
=n^{1 / 2} & \int_{0}^{\tau}\left[\hat{E} Q_{0}^{*}\left(\beta ; W_{i}, s\right) d N_{i}(s)\right. \\
& \left.-\left\{\frac{\hat{E} Q_{2}^{*}\left(\beta ; W_{i}, s\right)}{\hat{E} Q_{0}\left(\beta ; W_{i}, s\right)}-\frac{\hat{E} Q_{1}\left(\beta ; W_{i}, s\right) \hat{E} Q_{1}^{*^{T}}\left(\beta ; W_{i}, s\right)}{\hat{E}^{2} Q_{0}\left(\beta ; W_{i}, s\right)}\right\} \hat{E} d N(s)\right]+o_{p}(1),
\end{aligned}
$$

where

$$
\begin{aligned}
Q_{b i}^{*}\left(\beta ; s, W_{i}\right) & =Q_{b i}^{\#}\left(\beta ; W_{i}, s\right)\left(\hat{V}_{i}-V_{i}\right), \\
Q_{0 i}^{\#}\left(\beta ; W_{i}, s\right) & =\dot{g}\left(V_{i}, \boldsymbol{Z}_{i}, A_{i}\right), \\
Q_{1 i}^{\#}\left(\beta ; W_{i}, s\right) & =R_{i}(s) g\left(V_{i}, \boldsymbol{Z}_{i}, A_{i}\right) \exp \left\{\beta^{T} g\left(V_{i}, \boldsymbol{Z}_{i}, A_{i}\right)\right\} \dot{g}\left(V_{i}, \boldsymbol{Z}_{i}, A_{i}\right), \\
Q_{2 i}^{\#}\left(\beta ; W_{i}, s\right) & =R_{i}(s)\left\{I+g\left(V_{i}, \boldsymbol{Z}_{i}, A_{i} \dot{g}\left(V_{i}, \boldsymbol{Z}_{i}, A_{i}\right)\right) \beta\right\} \exp \left\{\beta^{T} g\left(V_{i}, \boldsymbol{Z}_{i}, A_{i}\right)\right\} .
\end{aligned}
$$

Using (B.7), we have

$$
n^{1 / 2} \hat{E} Q_{b}^{*}\left(\beta ; s, W_{i}\right)=\frac{1}{2} n^{-3 / 2} \sum_{i=1}^{n} \sum_{j=1}^{n} \eta_{b}\left(W_{i}, W_{j}, s\right)+o_{p}(1),
$$

where

$$
\begin{aligned}
\eta_{b}\left(\beta ; W_{i}, W_{j}, s\right)=\quad & Q_{b i}^{\#}\left(\beta ; W_{i}, s\right)\left[I\left(Y_{j}-\gamma_{0}^{T} \boldsymbol{Z}_{j} \leq Y_{i}-\gamma_{0}^{T} \boldsymbol{Z}_{i}\right)-h\left(Y_{i}-\gamma_{0}^{T} \boldsymbol{Z}_{i}\right)\right. \\
& \left.+h^{\prime}\left(Y_{i}-\gamma_{0}^{T} \boldsymbol{Z}_{i}\right)\left\{E(Z)-Z_{i}\right\}^{T}\left\{E\left(\boldsymbol{Z} \boldsymbol{Z}^{T}\right)\right\}^{-1} \boldsymbol{Z}_{j}\left(Y_{j}-\gamma_{0} \boldsymbol{Z}_{j}^{T}\right)\right] \\
+ & Q_{b i}^{\#}\left(\beta ; W_{j}, s\right)\left[I\left(Y_{i}-\gamma_{0}^{T} \boldsymbol{Z}_{i} \leq Y_{j}-\gamma_{0}^{T} \boldsymbol{Z}_{j}\right)-h\left(Y_{j}-\gamma_{0}^{T} \boldsymbol{Z}_{j}\right)\right. \\
& \left.+h^{\prime}\left(Y_{j}-\gamma_{0}^{T} \boldsymbol{Z}_{j}\right)\left\{E(Z)-Z_{j}\right\}^{T}\left\{E\left(\boldsymbol{Z} \boldsymbol{Z}^{T}\right)\right\}^{-1} \boldsymbol{Z}_{i}\left(Y_{i}-\gamma_{0}^{T} \boldsymbol{Z}_{i}\right)\right] .
\end{aligned}
$$

Note $E\left\{\eta_{b}\left(\beta ; W_{i}, W_{j}, s\right)\right\}=0$. It is simple to show that

$$
\frac{1}{2} n^{-3 / 2} \sum_{i=1}^{n} \sum_{j=1}^{n} \eta_{b}\left(\beta ; W_{i}, W_{j}, s\right)=\frac{2}{n(n-1)} \sum_{i=1}^{n} \sum_{j>1} \eta_{b}\left(\beta ; W_{i}, W_{j}, s\right)+o_{p}(1) .
$$


The first term on the right hand side is a U-process and can be rewritten as

$$
n^{1 / 2} \sum_{i=1}^{n} \eta_{b}^{*}\left(\beta ; W_{i}, s\right)+\frac{1}{n(n-1)} \sum_{i \neq j}^{n} \eta_{b}^{\#}\left(\beta ; W_{i}, W_{j}, s\right),
$$

where

$$
\begin{aligned}
\eta_{b}^{*}(\beta ; w, s) & =E\left\{\eta_{b}\left(\beta ; w, W_{2}, s\right)\right\}, \\
\eta_{b}^{\#}\left(\beta ; W_{i}, W_{j}, s\right) & =\eta_{b}\left(\beta ; W_{i}, W_{j}, s\right)-\eta_{b}^{*}\left(\beta ; W_{i}, s\right)-\eta_{b}^{*}\left(\beta ; W_{j}, s\right) .
\end{aligned}
$$

The second term in (B.8) is a degenerate U-process. Under conditions $\mathrm{E}^{\prime}$ and $\mathrm{G}^{\prime}$, the class of functions $\left\{\eta_{b}^{\#}(\beta, \cdot, \cdot, s): \beta \in \mathcal{N}\left(\beta_{0}\right), s \in[0, \tau]\right\}$ is Euclidean by Lemma 22(ii) in Nolan and Pollard (1987) and Lemma (2.14) in Pakes and Pollard (1989). With an application of Theorem 9 in Nolan and Pollard, the second term in (B.8) is equal to $o_{p}(1)$. Hence

$$
n^{1 / 2} \hat{E} Q_{b}^{*}\left(\beta ; s, V_{i}, \boldsymbol{Z}_{i}, A_{i}\right)=n^{1 / 2} \sum_{i=1}^{n} \eta_{b}^{*}\left(\beta ; W_{i}, s\right)+o_{p}(1) .
$$

Now using a functional Taylor expansion, we have

$$
n^{1 / 2}\{\hat{U}(\beta ; \hat{W})-\hat{U}(\beta ; W)\}=n^{-1 / 2} \sum_{i=1}^{n} \zeta\left(\beta ; W_{i}\right)+o_{p}(1)
$$

where

$$
\begin{aligned}
\zeta\left(\beta ; W_{i}\right)= & \int_{0}^{\tau}\left(\eta_{0}^{*}\left(\beta ; W_{i}, s\right) d N_{i}(s)\right. \\
& -\left[\frac{\eta_{2 i}^{*}\left(\beta ; W_{i}, s\right)}{E Q_{0}\left(\beta ; W_{i}, s\right)}-\frac{E \eta_{2}^{*}\left(\beta ; W_{i}, s\right)\left[Q_{0 i}\left(\beta ; W_{i}, s\right)-E\left\{Q_{0 i}\left(\beta ; W_{i}, s\right)\right\}\right]}{E^{2} Q_{0}\left(\beta ; W_{i}, s\right)}\right. \\
& -\frac{E \eta_{1}^{*^{T}}\left(\beta ; W_{i}, s\right)\left[Q_{1 i}\left(\beta ; W_{i}, s\right)-E Q_{1}\left(\beta ; W_{i}, s\right)\right]+E Q_{1}\left(\beta ; W_{i}, s\right) \eta_{1 i}^{*}\left(\beta ; W_{i}, s\right)}{E^{2} Q_{0}\left(\beta ; W_{i}, s\right)} \\
& \left.\left.+\frac{2 E Q_{1}\left(\beta ; W_{i}, s\right) E \eta_{1}^{*^{T}}\left(\beta ; W_{i}, s\right)\left[Q_{0 i}\left(\beta ; W_{i}, s\right)-E Q_{0}\left(\beta ; W_{i}, s\right)\right]}{E^{3} Q_{0}\left(\beta ; s, V_{i}, \boldsymbol{Z}_{i}, A_{i}\right)}\right] E d N(s)\right) .
\end{aligned}
$$

Also with a functional Taylor expansion, we have

$$
n^{1 / 2}\{\hat{U}(\beta ; W)-U(\beta)\}=n^{-1 / 2} \sum_{i=1}^{n} \rho\left(\beta ; W_{i}\right)+o_{p}(1) .
$$

Hence

$$
\begin{aligned}
& n^{1 / 2}\{\hat{U}(\beta ; \hat{W})-U(\beta)\} \\
= & n^{1 / 2}\{\hat{U}(\beta ; \hat{W})-\hat{U}(\beta ; W)\}+n^{1 / 2}\{\hat{U}(\beta ; W)-U(\beta)\} \\
= & n^{-1 / 2} \sum_{i=1}^{n}\left\{\zeta\left(\beta ; W_{i}\right)+\rho\left(\beta ; W_{i}\right)\right\}+o_{p}(1) .
\end{aligned}
$$


Then the consistency and asymptotical normality of $\tilde{\beta}$ follows by arguments similar to those in the appendix of Huang and Wang (2000), and we obtain the following asymptotic expansion:

$$
n^{1 / 2}\left\{\tilde{\beta}-\beta_{0}\right\}=n^{-1 / 2} \sum_{i=1}^{n} \pi^{*}\left(\beta_{0} ; W_{i}\right)+o_{p}(1)
$$

where $\pi^{*}\left(\beta_{0} ; W_{i}\right)=\Gamma^{-1}\left(\beta_{0}\right)\left\{\zeta\left(\beta ; W_{i}\right)+\rho\left(\beta ; W_{i}\right)\right\}$; that is, $n^{1 / 2}\left\{\tilde{\beta}-\beta_{0}\right\}$ is normal with mean zero and asymptotic variance $\operatorname{var}\left\{\pi^{*}\left(\beta_{0} ; W_{i}\right)\right\}$. The Breslow estimator for $\Lambda_{0}(t)$ can be written as

$$
\tilde{\Lambda}_{0}\left(t ; \hat{\beta}, \hat{W}_{i}\right)=\int_{0}^{t} \frac{\hat{E} d N(s)}{\hat{E} Q_{0}\left(\hat{\beta} ; \hat{W}_{i}, s\right)} .
$$

With similar arguments to those for $n^{1 / 2}\{\hat{U}(\beta ; \hat{W})-\hat{U}(\beta ; W)\}$, we can show that

$$
n^{1 / 2}\left\{\tilde{\Lambda}_{0}\left(t ; \beta, \hat{W}_{i}\right)-\tilde{\Lambda}_{0}\left(t ; \beta, W_{i}\right)\right\}=n^{-1 / 2} \sum_{i=1}^{n} \kappa(t ; \beta, W)+o_{p}(1)
$$

where

$$
\begin{aligned}
& \kappa(t ; \beta, W)= \\
& \quad-\int_{0}^{t}\left[\frac{\eta_{1}^{*}\left(\beta ; W_{i}, s\right)}{E^{2} Q_{0}(\beta ; W, s)}-\frac{2 E \eta_{1}^{*}(\beta ; W, s)\left\{Q_{0 i}\left(\beta ; W_{i}, s\right)-E Q_{0}\left(\beta ; W_{i}, s\right)\right\}}{\hat{E}^{3} Q_{0}\left(\beta ; W_{i}, s\right)}\right] E d N(s) .
\end{aligned}
$$

Also with a functional Taylor expansion, we have

$$
n^{1 / 2}\left\{\tilde{\Lambda}_{0}\left(t ; \beta, W_{i}\right)-\Lambda_{0}(t)\right\}=n^{-1 / 2} \sum_{i=1}^{n} \delta\left(t, \beta, W_{i}\right)+o_{p}(1) .
$$

Therefore

$$
\begin{aligned}
& n^{1 / 2}\left\{\tilde{\Lambda}_{0}\left(t ; \beta, \hat{W}_{i}\right)-\Lambda_{0}(t)\right\} \\
= & n^{1 / 2}\left\{\tilde{\Lambda}_{0}\left(t ; \beta, \hat{W}_{i}\right)-\tilde{\Lambda}_{0}\left(t ; \beta, W_{i}\right)\right\}+n^{1 / 2}\left\{\tilde{\Lambda}_{0}\left(t ; \beta, W_{i}\right)-\Lambda_{0}(t)\right\} \\
= & n^{-1 / 2} \sum_{i=1}^{n}\left(\kappa(\beta ; W)+\int_{0}^{t} \frac{d N_{i}(s)-R_{i}(s) \exp \left\{\beta_{0}^{T} g\left(V_{i}, \boldsymbol{Z}_{i}, A_{i}\right)\right\} E[d N(s)]}{E Q_{0}\left(\beta ; W_{i}, s\right)}\right)+o_{p}(1) .
\end{aligned}
$$

With a functional Taylor expansion, we have

$$
\begin{aligned}
& n^{1 / 2}\left\{\tilde{\Lambda}_{0}\left(t ; \tilde{\beta}, W_{i}\right)-\Lambda_{0}(t)\right\} \\
= & n^{-1 / 2} \sum_{i=1}^{n} \tilde{\delta}\left(t, \beta, W_{i}\right)-\int_{0}^{t} \frac{E Q_{1}\left(\beta ; W_{i}, s\right) E d N(s)}{E^{2} Q_{0}\left(\beta ; W_{i}, s\right)} n^{1 / 2}\left\{\tilde{\beta}-\beta_{0}\right\}+o_{p}(1),
\end{aligned}
$$

where

$$
\tilde{\delta}\left(t, \beta, W_{i}\right)=\left(\kappa(\beta ; W)+\int_{0}^{t} \frac{d N_{i}(s)-R_{i}(s) \exp \left\{\beta_{0}^{T} g\left(V_{i}, \boldsymbol{Z}_{i}, A_{i}\right)\right\} E[d N(s)]}{E Q_{0}\left(\beta ; W_{i}, s\right)}\right)
$$


Then using arguments similar to those for $\tilde{\theta}(v \mid \boldsymbol{z})$, we can show that

$$
n^{1 / 2}\left\{\tilde{\theta}^{*}(v \mid \boldsymbol{z})-\theta(v \mid \boldsymbol{z})\right\}=n^{1 / 2} \sum_{i=1}^{n} \mu^{*}(v ; \boldsymbol{z})+o_{p}(1)
$$

where

$$
\mu^{*}(v ; \boldsymbol{z})=\int_{v}^{1} \int_{0}^{L} \tilde{\xi}\left(\beta, t, u, \boldsymbol{z}, 1, X_{i}, \boldsymbol{Z}_{i}, A_{i}\right) d t d u+\int_{0}^{v} \int_{0}^{L} \tilde{\xi}\left(\beta, t, u, \boldsymbol{z}, 0, X_{i}, \boldsymbol{Z}_{i}, A_{i}\right) d t d u,
$$

where $\tilde{\xi}\left(\beta, t, u, \boldsymbol{z}, a, W_{i}\right)$ is obtained by replacing $\delta\left(t, \beta, W_{i}\right)$ by $\tilde{\delta}\left(t, \beta, W_{i}\right)$ and $\pi\left(\beta_{0} ; X_{i}, \boldsymbol{Z}_{i}, A_{i}\right)$ by $\pi^{*}\left(\beta_{0} ; X_{i}, Z_{i}, A_{i}\right)$, that is,

$$
\begin{aligned}
& \tilde{\xi}\left(\beta, t, u, \boldsymbol{z}, a, W_{i}\right)=S(t \mid u, \boldsymbol{z}, a) \exp \left\{\beta_{0}^{T} g(u, \boldsymbol{z}, a)\right\} \\
& \quad \times\left(\delta\left(t, \beta, W_{i}\right)\left\{\int_{0}^{t} \frac{E Q_{1}\left(\beta ; W_{i}, s\right) E d N(s)}{E^{2} Q_{0}\left(\hat{\beta} ; W_{i}, s\right)}-\Lambda_{0}(t) g(u, \boldsymbol{z}, a)\right\} \pi^{*}\left(\beta_{0} ; X_{i}, \boldsymbol{Z}_{i}, A_{i}\right)\right) .
\end{aligned}
$$

Hence $n^{1 / 2}\left\{\tilde{\theta}^{*}(v \mid \boldsymbol{z})-\theta(v \mid \boldsymbol{z})\right\}$ converges to a Gaussian process $\mathbb{G}^{\#}(v, \boldsymbol{z})$ with mean zero and covariance $\operatorname{cov}\left\{\mathbb{G}^{\#}\left(v_{1} ; \boldsymbol{z}\right), \mathbb{G}^{\#}\left(v_{2} ; \boldsymbol{z}\right)\right\}=\operatorname{cov}\left\{\mu^{*}\left(v_{1} ; \boldsymbol{z}\right), \mu^{*}\left(v_{2} ; \boldsymbol{z}\right)\right\}$ for $v_{1}, v_{2} \in(p, q)$.

\section{References}

Andersen, P.K. and Gill, R.D. (1982). Cox's Regression model for counting processes: a large sample study. Ann. Statist., 10, 1100-1120.

Augenlicht, L.H., Wadler, S., Corner, G., Richards, C., Ryan, L., Multani, A.S., Pathak, S., Benson, A., Hailer, D. and Heerdt B.G. (1997). Low-level c-myc amplification in human colonic carcinoma cell lines and tumors: a frequent, p53-independent mutation associated with improved outcome in a randomized multi-institutional trial. Cancer Research, 57, 1769-1775.

Chen, P.Y. and Tsitais, A.A. (2001). Causal inference on the difference of the restricted mean lifetime between two groups. Biometrics, 57, 1030-1038.

Elmer-Dewitt, P., Lemonick, M., Park, A., and Nash, M. (2001). Medicine: the future of drugs. Time, 157, 56-102.

Erisman, M.D., Scott, J.K., Watt, R.A., and Astrin, S.M. (1988) The c-myc protein is constitutively expressed at elevated levels in colorectal carcinoma cell lines. Oncogene, 2, 367-378.

Heagerty P.J. and Pepe. M.S. (1999). Semiparametric estimation of regression quantiles with application to standarizing weight for height and age in U.S. children. Appl. Statistics, 48, 533-551.

Holland, P. (1986). Statistics and causal inference. J. Am. Statist. Ass. 81, 945-960.

Huang, Y. and Wang, C.Y. (2000). Cox regresson with acurate covariats unascertainable: a nonparametric-correciton approach. Journal of the American Statistical Association 95, $1209-1219$. 
Klein J.P. and Moeschberger M.L. (2003). Survival Analysis: Techniques for Censored and Truncated Data. Springer: New York.

Li, Y. and Ryan, L. (2006). Inference on survival data with covariate measurement error an imputation-based approach. Scandinavian Journal of Statistics, 33, 169-190.

Pepe, M.S. (1998). Three approaches to regression analysis of receiver operating characteristic curves in medical diagnostic testing. Biometrics, 54, 124-135.

Pepe, M.S. (2000). Receiver operating characteristic methodology. J. Am. Statist. Ass., 95, 308-311.

Pepe, M.S. (2003). The Statistical Evaluation of Medical Tests for Classification and Prediction. Oxford University Press.

Rubin, D.B. (1974). Estimating causal effects of treatments in randomized and nonrandomized studies. Journal of Educational Psychology, 66, 688-701.

Rubin, D.B (1978). Bayesian inference for causal effects: The role of randomization. Ann. Statist. 6, 34-58.

Song, X., Ma, S., Huang, J. and Zhou, X.H. (2007). A semiparametric approach for the nonparametric transformation survival model with multiple covariates. Biostatistics, 8, $197-211$.

Song X. and Pepe M.S. (2004). Evaluating markers for selecting a patient's treatment. Biometrics, 60, 874-883.

Van der Vaart, A.W. and Wellner, J.A. (2000). Weak Convergence and Empirical Processes. New York: Springer-Verlag.

Zhao, H. and Tsitais, A.A. (1997). A consistent estimator for the distribution of quality adjusted lifetime. Biometrika, 84. 339-348.

Zhou, X-H., Obuchowski, N.A. and McClish, D.K. (2002). Statistical Methods in Diagnostic Medicine. New York:Wiley. 


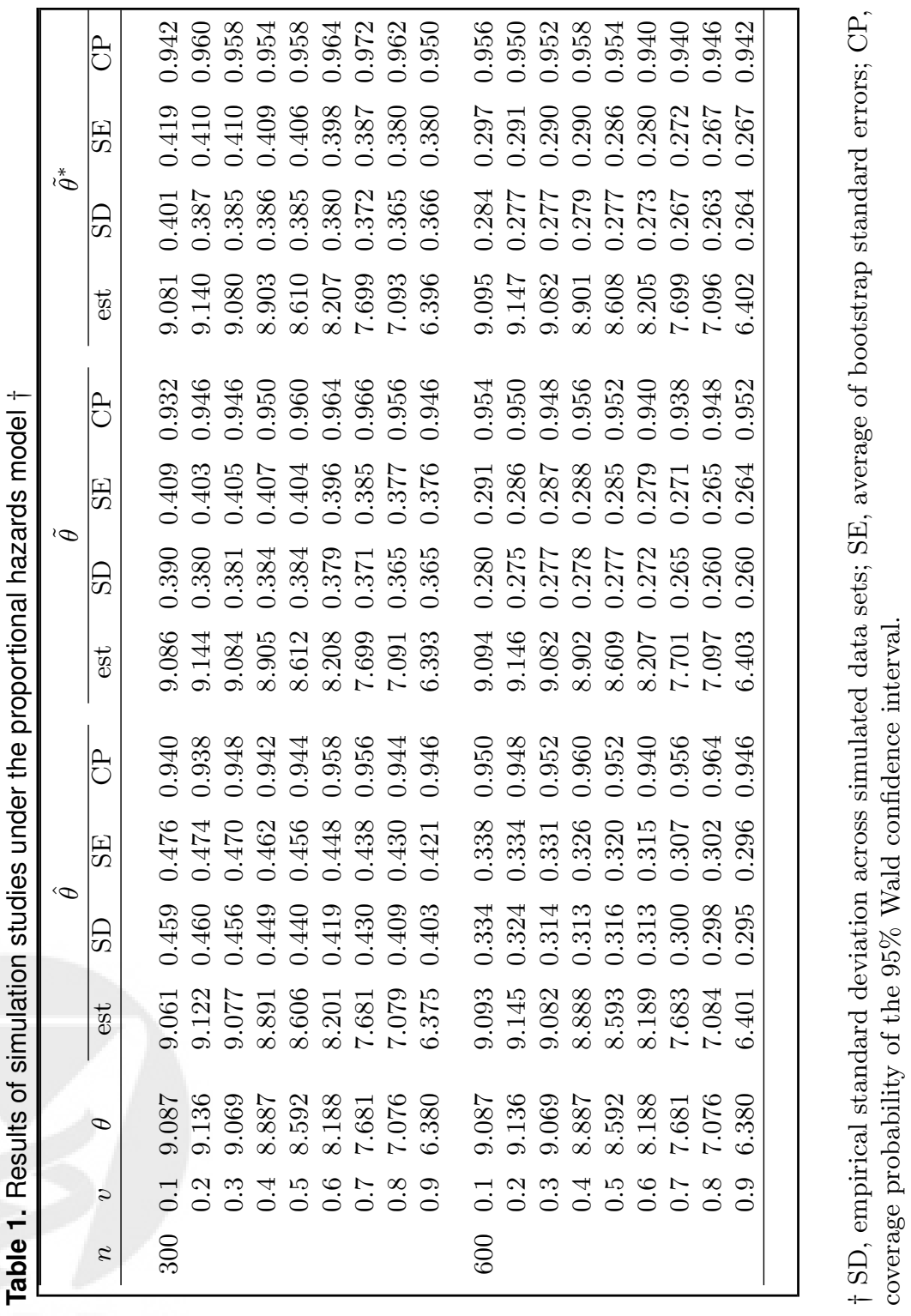




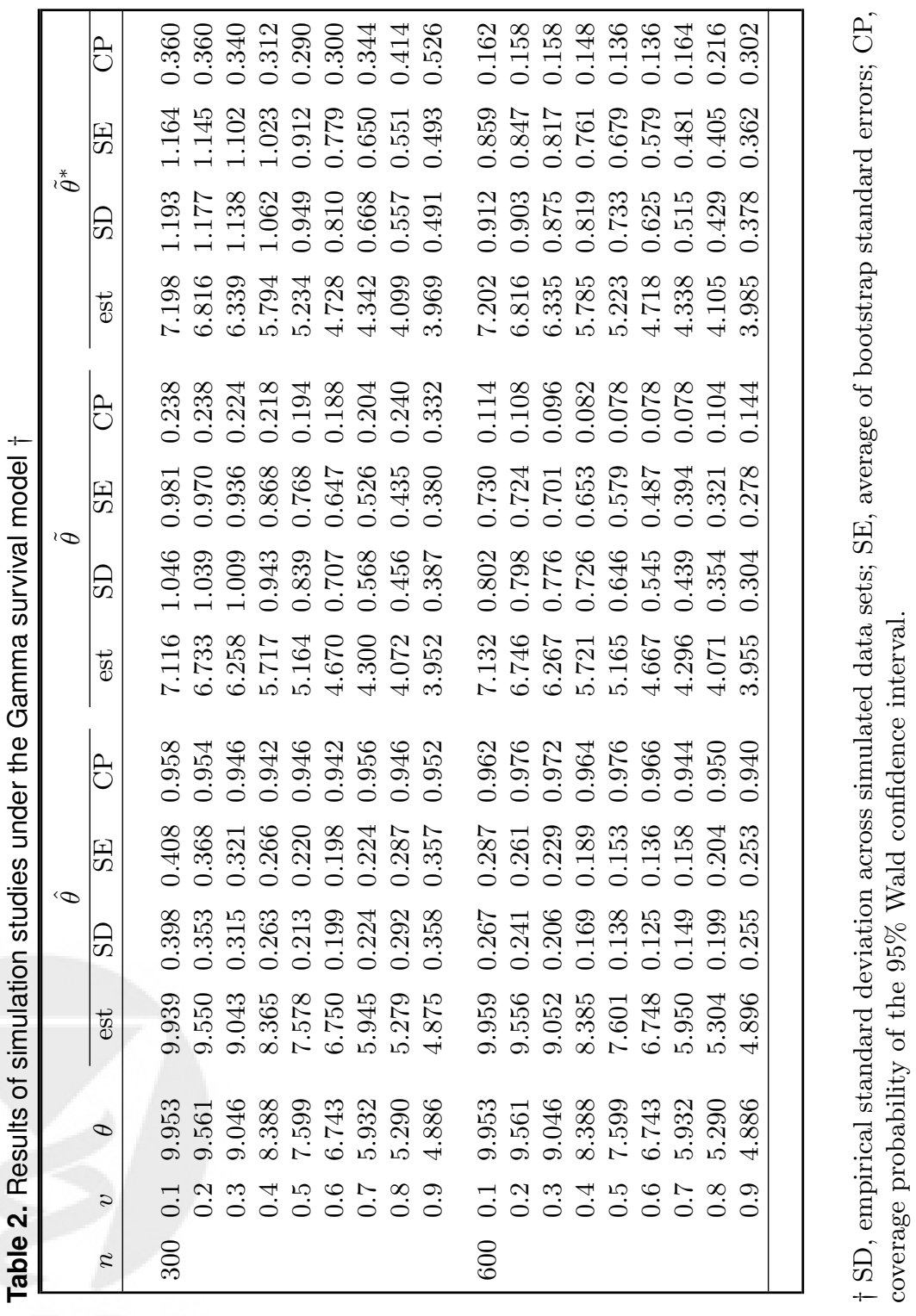




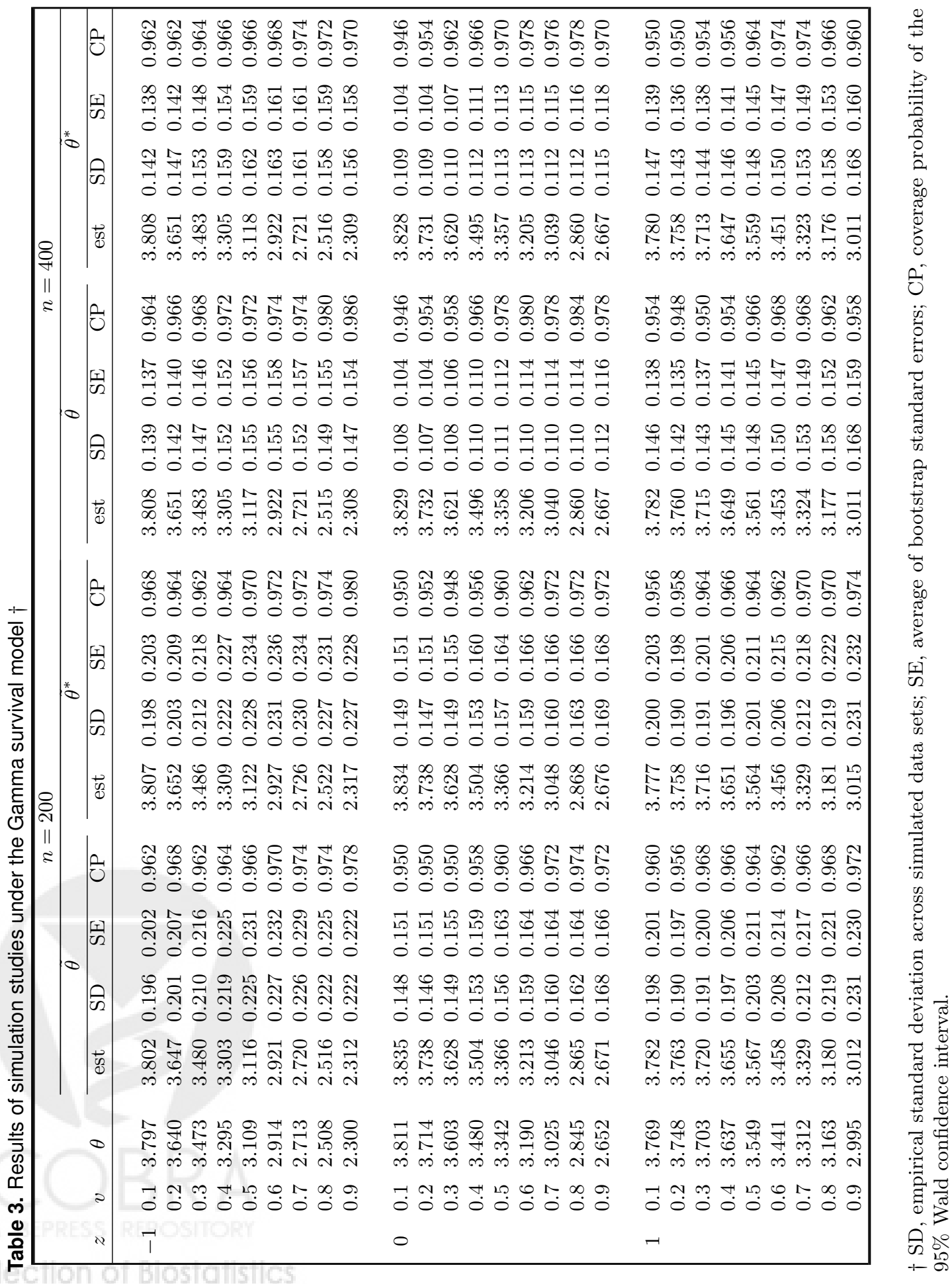




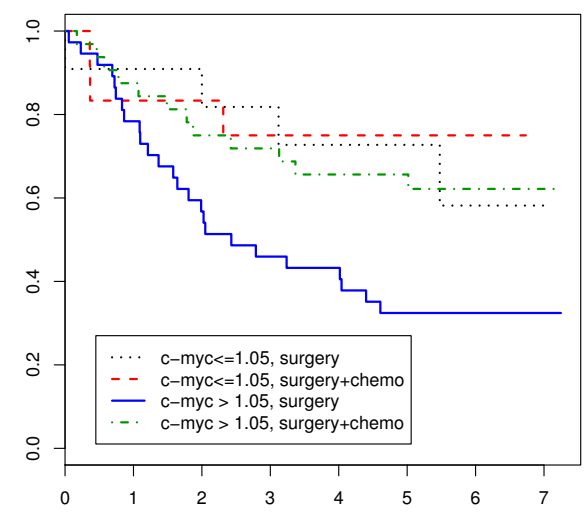

Fig. 1. Kaplan-Meier estimates of disease progression free survival by treatment and c-myc level

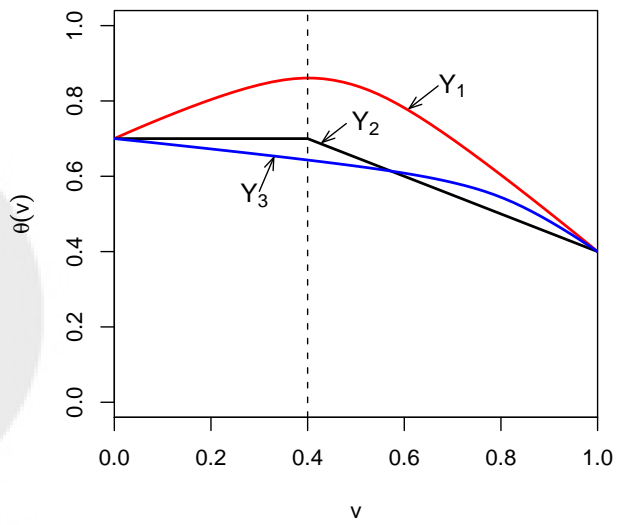

Fig. 2. Example of SI curves 


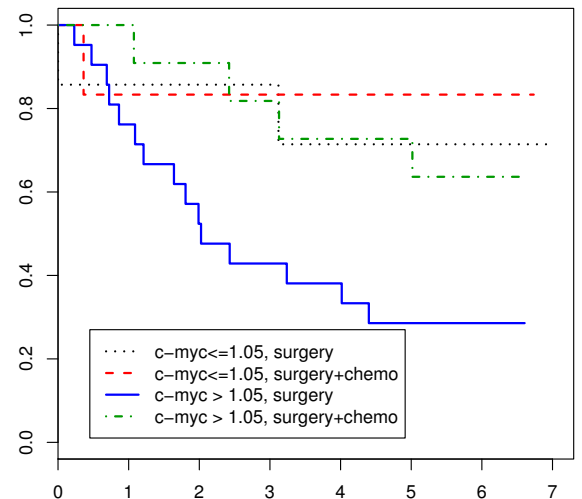

(a) Female

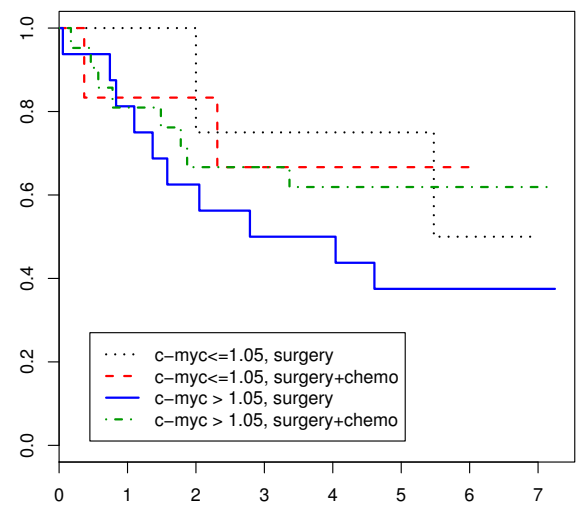

(b) Male

Fig. 3. Kaplan-Meier estimates of disease progression free survival by treatment and c-myc level for different genders.

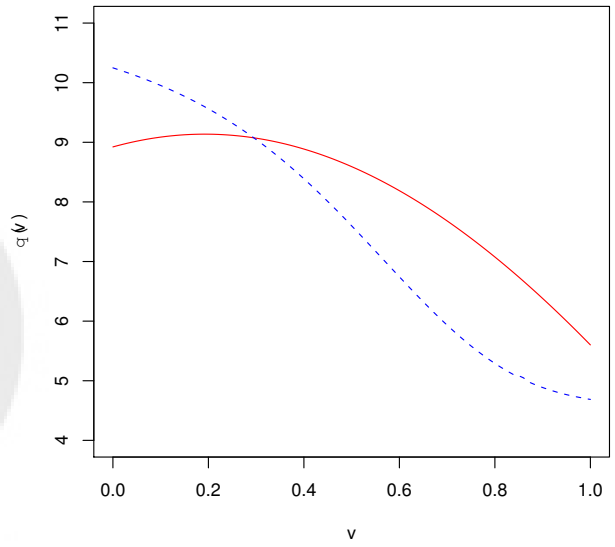

Fig. 4. True SI curve in simulation. Solid line, proportional hazards modal; dashed line, Gamma survival distribution. 


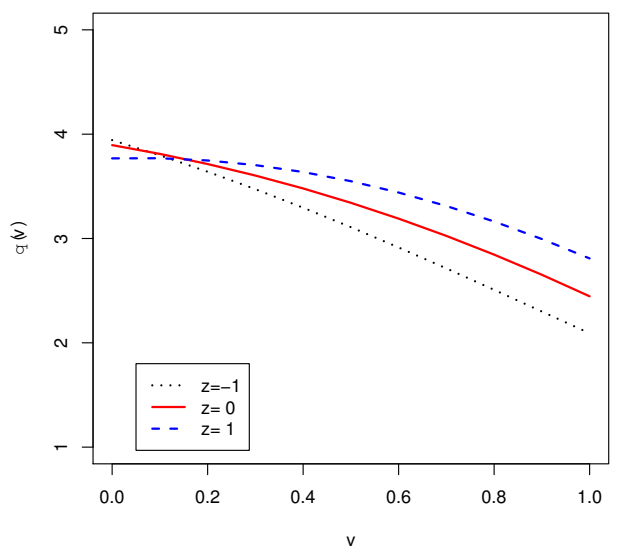

Fig. 5. True covariate specific SI curve in simulation.
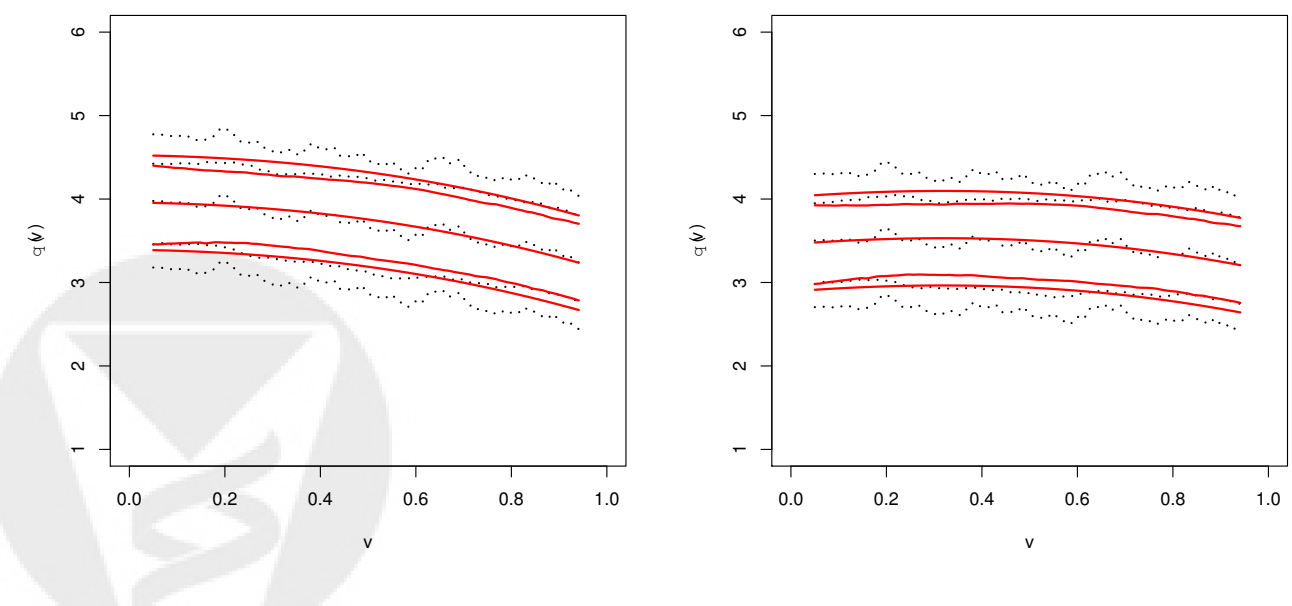

Fig. 6. Estimated SI curve for ECOG data. Left panel is based on 5 year restricted survival time $T^{*}$, right panel is based on the utility function $U=T^{*}-0.5 A$. Nonparametric estimate $\hat{\theta}$, solid curve; semiparametric estimate $\tilde{\theta}$, dotted curve. $95 \%$ confidence bands are shown with the outer curves, $95 \%$ pointwise confidence intervals are shown with the intermediate curves, the estimates themselves are shown with the center curves. 

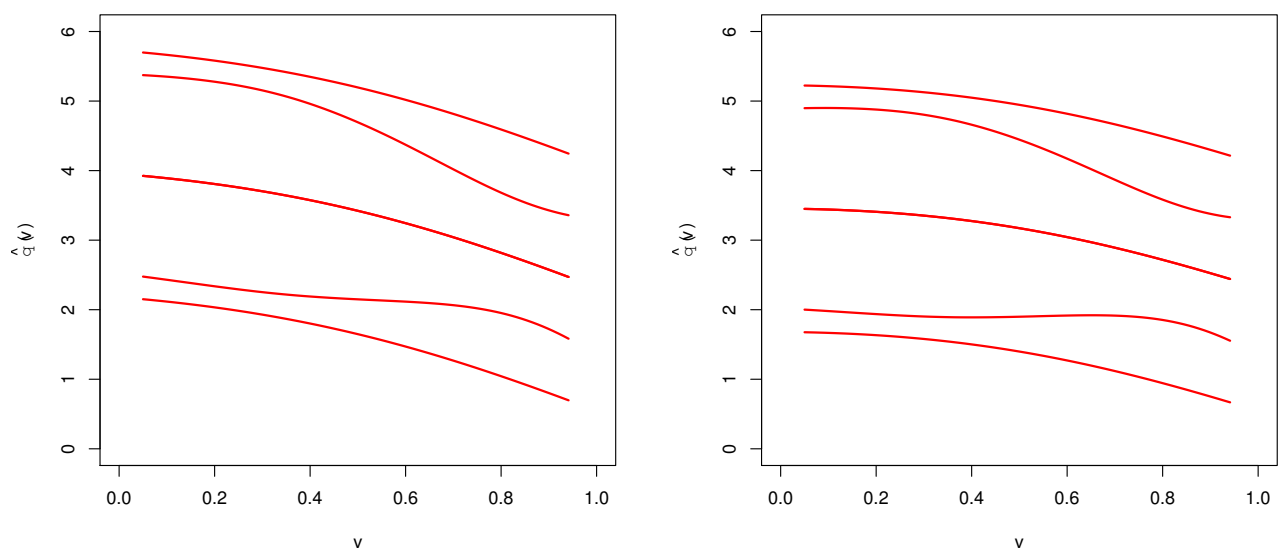

(a) Female
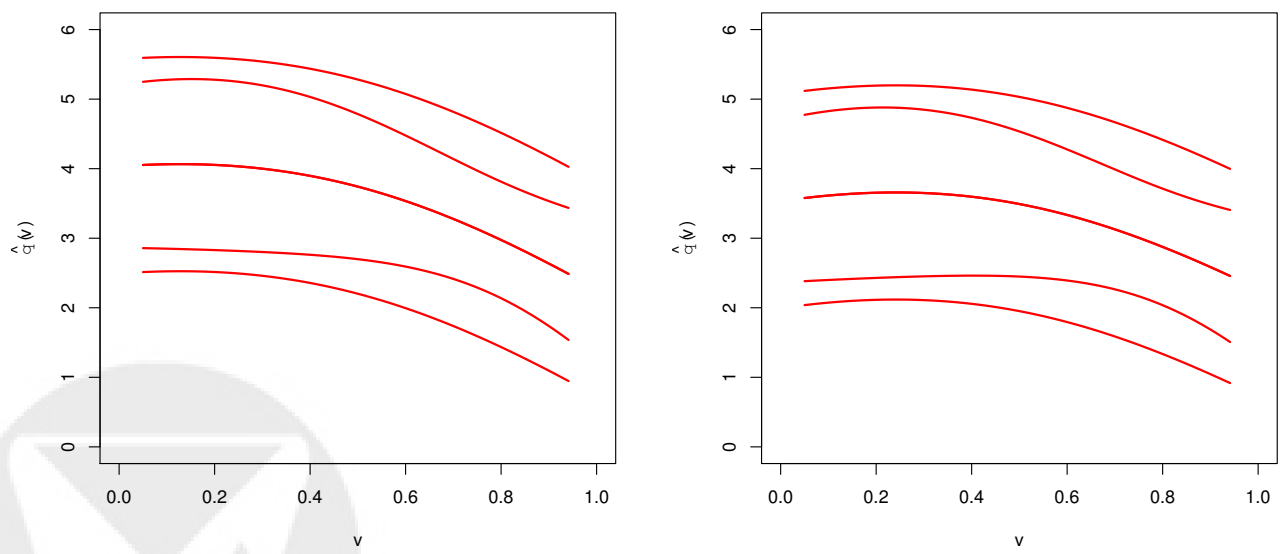

(b) Male

Fig. 7. Estimated SI curves adjusted for gender for ECOG data. Left panel is based on 5 year restricted survival time $T^{*}$, right panel is based on the utility function $U=T^{*}-0.5 \mathrm{~A}$. 95\% confidence bands are shown with the outer curves, 95\% pointwise confidence intervals are shown with the intermediate curves, the estimates themselves are shown with the center curves. 